\title{
On a Form of the First Variation of the Action Integral Over a Varied Domain
}

\author{
V. A. Kovalev ${ }^{1}$, Yu. N. Radayev ${ }^{2}$ \\ ${ }^{1}$ Moscow City Government University of Management, 28, Sretenka str., 107045, Moscow, Russia, kovalev.kam @ gmail.com \\ ${ }^{2}$ Institute for Problems in Mechanics of RAS, 101-1, Vernadskogo ave., 119526, Moscow, Russia, radayev@ipmnet.ru, \\ y.radayev@gmail.com
}

Field theories of the continuum mechanics and physics based on the least action principle are considered in a unified framework. Variation of the action integral in the least action principle corresponds variations of physical fields while space-time coordinates are not varied. However notion of the action invariance, theory of variational symmetries of action and conservation laws require a wider variation procedure including variations of the space-time coordinates. A similar situation is concerned to variational problems with strong discontinuities of field variables or other a priori unknown free boundaries which variations are not prohibited from the beginning. A form of the first variation of the action integral corresponding variations of space - time coordinates and field variables under one-parametrical transformations groups is obtained. This form is attributed to 4-dimensional covariant formulations of field theories of the continuum mechanics and physics. The first variation of the action integral over a varied domain is given for problems with constraints. The latter are formulated on unknown free boundaries.

Key words: field, action, least action principle, field equations, transformation group, Lie group, infinitesimal generator, variation, varied domain, constraint.

This work was supported by the Russian Foundation for Basic Research (project no. 13-01-00139).

\section{References}

1. Kovalev V. A., Radayev Yu. N. Elements of the Field Theory: Variational Symmetries and Geometric Invariants. Moscow, Fizmatlit, 2009, 156 p. (in Russian). 2. Kovalev V. A., Radayev Yu. N. Wave Problems of Field Theory and Thermomechanics. Saratov, Saratov Univ. Press, 2010. 328 p. (in Russian).

3. Ovsyannikov L. V. Group Analysis of Differential Equations. Moscow, Nauka, 1978, 400 p. (in Russian).

4. Olver P. J. Applications of Lie Groups to Differential Equations. Moscow, Mir, 1989, 639 p. (in Russian).

5. Olver P. J. Equivalence, Invariants and Symmetry. Cambridge ; N.Y. ; Melbourne, Cambridge University Press, 1995, 526 p.

6. Noether E. Invariante Variationsprobleme. Nachrichten von der Königlichen Gesellschaft der Wissenschaften zu Göttingen. Mathematisch-Physikalische Klasse, 1918, H. 2, s. 235-257. (Rus. ed. : Noether E. Invariantnye variatsionnye zadachi // Variatsionnye printsipy mekhaniki. Moscow, Fizmatgiz, 1959, pp. 611630).

7. Courant R., Gilbert D. Methods of Mathematical Physics : in 2 vol. Moscow; Leningrad, Gostekhteoretizdat, 1933, vol. 1, 528 p. (in Russian).

8. Gelfand I. M., Fomin S. V. Calculus of Variations. Moscow, Fizmatgiz, 1961, 228 p. (in Russian).

9. Gunter N. M. A Cours of the Calculus of Variations. Moscow; Leningrad, Gostekhteoretizdat, 1941, 308 p. (in Russian).

УДК 539.3

\section{ОСЕСИММЕТРИЧНОЕ НАРАЩИВАНИЕ ПОЛОГО ГИПЕРУПРУГОГО ЦИЛИНДРА}

\author{
С. А. Лычев ${ }^{1}$, А. В. Марк ${ }^{2}$
}

\begin{abstract}
${ }^{1}$ Доктор фризико-математических наук, ведущий научный сотрудник лаборатории моделирования в механике десормируемого твердого тела, Институт проблем механики им. А. Ю. Ишлинского РАН, Москва, lychevsa@ mail.ru

${ }^{2}$ Кандидат фризико-математических наук, младший научный сотрудник лаборатории моделирования в механике десрормируемого твердого тела, Институт проблем механики им. А. Ю. Ишлинского РАH, Mockва, A-V-Mark@yandex.ru
\end{abstract}

Исследуется напряженно-десрормированное состояние растущего цилиндра из несжимаемого упругого материала типа Муни - Ривлина при конечных десрормациях. Десормации полагаются осесимметричными и не изменяющимися вдоль оси цилиндра. Рассмотрены дискретные и непрерывные режимы наращивания. Построены соответствующие решения краевых задач. На основе вычислений показана сходимость решения для дискретного наращивания к решениям задач для непрерывного наращивания при увеличении количества слоев и уменьшения их толщины в условиях фриксированного фринального объема.

Ключевые слова: аддитивные технологии, растущие тела, конечные десрормации, гиперупругость, непрерывное наращивание, дискретное наращивание. 


\section{ВВЕДЕНИЕ}

При моделировании разнообразных аддитивных технологических процессов [1] необходимо учитывать механические эффекты, сопутствующие процессам соединения деформируемых частиц в единое тело или, иначе говоря, вызванные ростом деформируемого тела. Эти эффекты проявляют себя как поля остаточных напряжений и как связанные с ними отклонения финальной формы изделия от проектной. В качестве примеров могут быть приведены технологии стереолитографии, трехмерной печати, парофазного осаждения, наплавки и т.п. [1-3]. При разработке технологий подобных процессов зачастую желательно минимизировать искажения и остаточные напряжения, либо создавать конструкции с заранее заданными распределениями начальных напряжений. Этого можно достичь посредством математического моделирования эволюции напряженно-деформированного состояния растущего тела, вопросам которого посвящена настоящая статья.

В настоящей работе используется понятие роста деформируемого твердого тела. Само это понятие относится к новому направлению - механике растущих тел [4-7], в связи с чем представляется здесь уместным уточнить его определение. Рост в широком смысле определяется изменением материального состава тела, происходящим в процессе его деформирования, которое может сопровождаться изменением его топологических свойств. Под изменением материального состава понимается присоединение новых материальных точек и (или) образование новых связей между материальными точками, уже входящими в состав тела. Необходимо отметить, что изменение топологических свойств может происходить без притока материала извне и может быть вызвано переходом части граничных точек во внутренность тела. Классическими примерами здесь являются склеивание берегов разреза кольца с вырезанным сектором и преобразование конечного цилиндра в полноторие с отождествлением его оснований, либо отождествлением сечений полнотория с удалением сектора, заключенного между ними (этот пример иллюстрирует понятие дислокации и был впервые использован В. Вольтерра [8]). С позиций, принимаемых в рамках настоящей работы, подобные преобразования следует рассматривать как специальные варианты дискретного роста.

В современной механике континуума сформировался ряд различных подходов к изучению феномена роста. К настоящему времени опубликовано большое количество работ, посвященных механике растущих тел и иллюстрирующих эти подходы. Ссылки на многие из них можно найти в обзоре [9]. Здесь укажем лишь некоторые работы, имеющие непосредственное отношение к вопросам, затрагиваемым в статье. В исследованиях [10] рассматривается объемный рост, в частности, рост биологических тканей. Статья [11] посвящена развитию геометрических методов механики к описанию несовместных деформаций, возникающих как результат процесса роста. В работах [4-7, 12] исследуется поверхностный рост как процесс непрерывного присоединения напряженных материальных поверхностей к растущему деформируемому телу.

Известно, что при дополнительных предположениях о непрерывности функций, определяющих изменение напряженно-деформируемого состояния присоединяемых материальных поверхностей, непрерывный процесс можно рассматривать как предел последовательности процессов дискретного наращивания при неограниченном уменьшении толщин присоединяемых слоев. Вместе с тем, в литературе представлены лишь немногочисленные примеры исследования напряженно-деформированного состояния растущих тел при конечных деформациях. Настоящая статья частично восполняет этот пробел. В ней рассматривается решение модельной задачи о растущем цилиндре, которое иллюстрирует качественные особенности процессов дискретного и непрерывного наращивания. Показана сходимость полученных решений для дискретного наращивания к решениям соответствующих задач для непрерывного наращивания при увеличении количества слоев и уменьшения их толщины в условиях фиксированного финального объема.

\section{1. ОБЩИЕ ОПРЕДЕЛЕНИЯ}

Для построения математической модели растущего тела воспользуемся формализацией тела как гладкого многообразия, которая развита в работах школы рациональной механики [13-17]. В рамках этой аксиоматики под телом $\mathscr{B}$ будем понимать связное подмножество абстрактного топологического пространства, такое, что его образ в физическом пространстве представляет собой область с регулярной границей. Кроме того, полагаем, что тело представляет собой дифференцируемое много- 
образие [18, 19]. Его элементы $\mathfrak{p} \in \mathscr{B}-$ материальные точки; полагаем, что они являются простыми, т. е. тело состоит из простого материала, в котором напряжения в некоторой точке зависят только от первого градиента места в окрестности этой точки [20].

В классической механике сплошных сред тела рассматриваются как фиксированные множества материальных точек. В задачах механики растущих тел рассматривается эволюция множества $\mathscr{B}$, которое представляет собой множество переменного состава. Заметим, что эволюция тела как точечного множества в абстрактном топологическом (материальном) пространстве может быть весьма сложной [21]. Однако при некоторых ограничениях на гладкость отображений, характеризующих процесс роста, ее можно представить непрерывным семейством тел, упорядоченных относительно вложения. В общем случае это семейство можно описать как расслоение гладкого многообразия [4], размерность базы которого определяет класс растущего тела. В настоящей работе будет рассмотрен наиболее простой класс, соответствующий одномерной базе расслоения. При этом рост в терминах механики деформируемого твердого тела характеризуется как непрерывный процесс присоединения к растущему твердому телу предварительно напряженных материальных поверхностей [22], и растущее тело может быть представлено однопараметрическим семейством гладких тел:

$$
\mathfrak{C}=\left\{\mathscr{B}_{\alpha}\right\}_{\alpha \in \mathscr{I}},
$$

где $\mathscr{I}-$ множество индексов, которое может быть конечным или континуальным.

Введем понятия тотального тела $\mathscr{B}^{*}$ и начального тела $\mathscr{B}_{*}$ следующим образом:

$$
\mathscr{B}^{*}=\bigcup_{\alpha \in \mathscr{I}} \mathscr{B}_{\alpha}, \quad \mathscr{B}_{*}=\bigcap_{\alpha \in \mathscr{I}} \mathscr{B}_{\alpha} .
$$

Элементы множества $\mathfrak{C}$, соответствующие внутренним точкам интервала $\mathscr{I}$, будем называть промежуточными телами.

Будем различать дискретный и непрерывный рост. В случае дискретного роста множество $\mathfrak{C}$ представляет собой конечную последовательность вложенных множеств:

$$
\mathfrak{C}: \mathscr{B}_{1} \subset \mathscr{B}_{2} \subset \ldots \subset \mathscr{B}_{N}
$$

В случае непрерывного роста множество $\mathfrak{C}-$ непрерывное семейство тел над интервалом $\mathscr{I}=(\alpha, \beta) \subset \mathbb{R}$, удовлетворяющее следующему условию: существуют двумерные гладкие многообразия $\Omega_{k}$ и не более чем счетное множество гомеоморфизмов ${ }^{1}$ :

$$
\Psi_{k}:\left(\Omega_{k}, \alpha\right) \rightarrow \mathscr{B}^{*}, \quad \alpha \in \mathbb{R},
$$

таких, что

$$
\forall \alpha<\beta \quad \mathscr{B}_{\alpha} \subset \mathscr{B}_{\beta}, \quad \forall \alpha \exists k \partial \mathscr{B}_{\alpha}=\Psi_{k}\left(\Omega_{k}, \alpha\right), \quad \bigcup_{k} \Psi_{k}\left(\Omega_{k} \times \mathscr{I}_{k}\right)=\mathscr{B}^{*}, \quad \bigcup_{k} \mathscr{I}_{k}=\mathscr{I} .
$$

Очевидно, что множество $\mathfrak{C}$ имеет счетную мощность $|\mathfrak{C}|=\aleph_{1}$. Многообразия $\Omega_{k}$ будем называть прообразом границы роста. Соотношения (2) устанавливают структуру гладкого расслоения многообразия $\mathscr{B}^{*}[4,18,19]$. При этом интервал $\mathscr{I}$ представляет собой базу расслоения, а многообразие $\Omega_{k}$ - прообраз слоя. Если существует единственный (универсальный) гомеоморфизм $\Psi=\Psi_{1}$ единственного многообразия $\Omega=\Omega_{1}$ на все границы элементов множества $\mathfrak{C}$, то расслоение оказывается тривиальным. В противном случае, топологическая структура прообраза растущей границы может меняться. Изменение топологии прообраза границы роста соответствует самосоприкосновению частей образа $\Omega_{k}$, например, преобразованию конечного цилиндра в полноторие при отождествлении его оснований.

Таким образом, растущее тело может быть представлено как расслоение тотального тела $\mathscr{B}^{*}$. Слои, соответствующие координате базы $\alpha$, будем обозначать символом $\mathfrak{M}_{\alpha}$. Из структурных свойств расслоения вытекает, что слои не пересекаются, а их объединение совпадает с тотальным телом $\mathscr{B}^{*}$, т. е.

\footnotetext{
${ }^{1}$ Гомеоморфизм - это непрерывное отображение топологических пространств $X \rightarrow Y$, которое имеет непрерывное обратное отображение. Отображение топологических пространств $X \rightarrow Y$ непрерывно, если для каждого открытого подмножества $V \subset Y$ его прообраз в $X$ открыт [19].
} 
$\mathscr{B}^{*}=\bigcup_{\gamma \in \mathscr{I}} \mathfrak{M}_{\gamma}$. В процессе роста тело $\mathscr{B}_{\alpha}$ представляется открытыми подмножествами тотального тела $\mathscr{B}^{*}$, граница которых $\partial \mathscr{B}_{\alpha}$ образуется двумя различными слоями $\mathfrak{M}_{\alpha^{\prime}}$ и $\mathfrak{M}_{\beta^{\prime}}$, т. е. $\partial \mathscr{B}_{\alpha}=\mathfrak{M}_{\alpha^{\prime}} \cup \mathfrak{M}_{\beta^{\prime}}$. В этом случае тело $\mathscr{B}_{\alpha}$ может быть представлено как объединение множества слоев, индексы которых принадлежат открытому интервалу $\left(\alpha^{\prime}, \beta^{\prime}\right) \subset \mathscr{I}$ :

$$
\mathscr{B}_{\alpha}=\mathscr{B}\left(\alpha^{\prime}, \beta^{\prime}\right)=\bigcup_{\gamma \in\left(\alpha^{\prime}, \beta^{\prime}\right)} \mathfrak{M}_{\gamma} .
$$

При принятых предположениях растущее тело определяется как однопараметрическое семейство таких множеств

$$
\mathfrak{C}=\left\{\mathscr{B}_{\alpha}=\mathscr{B}\left(\alpha_{0}, \alpha\right) \mid \alpha \in \mathscr{I}\right\}
$$

где $\alpha$ теперь определяет параметр семейства, характеризующий эволюцию растущего тела, причем при $\alpha \rightarrow \alpha_{0}$ тело вырождается в бесконечно тонкий слой. Очевидно обобщение этого определения

$$
\mathfrak{C}=\left\{\mathscr{B}_{\gamma}=\mathscr{B}\left(\alpha_{\gamma}, \beta_{\gamma}\right) \mid\left(\alpha_{\gamma}, \beta_{\gamma}\right) \subset \mathscr{I}\right\}
$$

где $\left(\alpha_{\gamma}, \beta_{\gamma}\right)-$ семейство вложенных интервалов.

Согласно данным определениям граница растущего тела должна быть топологически эквивалентна типовому слою, который сам представляет собой гладкое многообразие, и, следовательно, растущая граница топологически эквивалентна геометрически замкнутой поверхности. Если растущая граница топологически эквивалентна многообразию с краем, то растущее тело может быть определено следующим образом:

$$
\mathfrak{C}=\left\{\mathscr{B}_{\gamma}=\mathscr{B}_{0} \cap \mathscr{B}\left(\alpha_{\gamma}, \beta_{\gamma}\right) \mid\left(\alpha_{\gamma}, \beta_{\gamma}\right) \subset \mathscr{I}\right\}
$$

Здесь $\mathscr{B}_{0}$ - фиксированное подмножество материального многообразия с гладкой границей.

В настоящей работе рассматривается наращивание полого цилиндра фиксированной высоты, в ходе которого материал присоединяется к его боковой поверхности. Если на материальном многообразии ввести координатные карты, соответствующие положениям точек в какой-либо актуальной конфигурации, то карта для $\mathscr{B}_{0}$ будет соответствовать достаточно большому параллелепипеду, высота которого равна высоте растущего цилиндра.

\section{2. НАПРЯЖЕННО-ДЕФОРМИРОВАННОЕ СОСТОЯНИЕ ТЕЛА-СЛОЯ}

Напряженно-деформированное состояние (НДС) растущего тела принципиально отличается от НДС тел, рассматриваемых в классической механике деформируемого твердого тела тем, что растущее тело не обладает натуральной (свободной от напряжений) конфигурацией. Никакая его деформация не приводит к одновременной релаксации напряжений во всем теле. НДС таких тел может быть описано в рамках теории неоднородных, но материально единообразных тел, развитой в работах [13-15]. Представление тела как расслоение гладкого многообразия позволяет ввести гипотезы о свойствах составляющих его слоев, в частности, гипотезу о том, что каждый слой в отдельности обладает натуральной конфигурацией. Эта гипотеза принимается в настоящей работе.

Для описания НДС тела, растущего дискретным или непрерывным образом, необходимо определить НДС слоя как его составного элемента. В первом случае этот элемент представляет собой трехмерное тело $\mathscr{B}_{n+1} \backslash \mathscr{B}_{n}$, соответствующую инкременту последовательности (1), во втором случае - материальную поверхность $\mathfrak{M}_{\gamma}$, соответствующую слою расслоения (3). В рамках настоящей работы будем полагать, что тело-слой в отдельности обладает натуральной конфигурацией, погружаемой в евклидово пространство, но в совокупности слои не обладают таковой. Как уже отмечалось, в качестве модельного объекта, представляющего растущее тело, рассмотрим полый конечный цилиндр. Соединение элементов дискретного множества $\mathscr{B}_{N}$ и континуального семейства $\mathscr{B}_{\gamma}$, определяющее состав растущего тела, будем называть сборками, что образно отражает характер процесса как при дискретном, так и при непрерывном росте.

В случае дискретного роста каждая сборка состоит из конечного числа вложенных полых цилиндрических слоев. Второй случай, конечно, более абстрактный: он соответствует сборке континуального множества слоев, каждый из которых представлен двумерным телом - материальной 
поверхностью. Напряженное состояние, ассоциированное с материальной поверхностью, полагается реализуемым в составе некоторого тела, деформируемого из натурального состояния в евклидовом пространстве.

Будем полагать, что цилиндрические слои изготовлены из гиперупругого несжимаемого материала. Тогда их НДС может быть определено аналитически с помощью решений типа РивлинаЭриксена [20]. Кратко остановимся на форме этого решения.

Пусть в образе свободной от напряжений (натуральной) конфигурации положения точки $\mathfrak{p} \in \mathscr{B}_{n+1} \backslash \mathscr{B}_{n}$ тела-слоя с номером $n$ в физическом (евклидовом) пространстве $\mathscr{E}$ задаются векторным полем мест, которое может быть представлено в декартовом базисе $\left\{\boldsymbol{i}_{1}, \boldsymbol{i}_{2}, \boldsymbol{i}_{3}\right\}$ разложением $\boldsymbol{X}=X^{m} \boldsymbol{i}_{m}$, где $\left\{X^{1}, X^{2}, X^{3}\right\}$ - декартовы координаты точки. Пусть деформация слоя в элемент сборки определяется отображением $\boldsymbol{X} \mapsto \boldsymbol{x}$. Будем полагать, что это отображение обладает симметрией относительно общей оси цилиндрических слоев и не зависит от координаты $X^{3}$.

Для более компактной формулировки кинематических соотношений, определяющих деформацию, воспользуемся цилиндрическими координатами $\{R, \Theta, Z\}$ :

$$
X^{1}=R \cos \Theta, \quad X^{2}=R \sin \Theta, \quad X^{3}=Z, \quad R=\sqrt{\left(X^{1}\right)^{2}+\left(X^{2}\right)^{2}}
$$

В каждой точке $\mathscr{E}$ цилиндрические координаты определяют локальный базис $\left\{\boldsymbol{e}_{R}, \boldsymbol{e}_{\Theta}, \boldsymbol{e}_{Z}\right\}$ и взаимный к нему базис $\left\{\boldsymbol{e}^{R}, \boldsymbol{e}^{\Theta}, \boldsymbol{e}^{Z}\right\}$. Элементы базисов могут быть представлены разложениями

$$
e_{R}=e^{R}=i_{1} \cos \Theta+i_{2} \sin \Theta, \quad e_{\Theta}=-i_{1} R \sin \Theta+i_{2} R \cos \Theta, \quad e^{\Theta}=\frac{e_{\Theta}}{R^{2}}, \quad e^{Z}=e_{Z}=i_{3} .
$$

Представление отсчетных позиций точек цилиндрических слоев наиболее компактно выражается в форме разложения $\boldsymbol{X}=R \boldsymbol{e}_{R}+Z \boldsymbol{e}_{Z}$. Учитывая центральную симметрию смещений, их независимость от вертикальной координаты $Z$ и условие несжимаемости $|d \boldsymbol{x} / d \boldsymbol{X}|=1$, приходим к следующему классу отображений (универсальных деформаций, принадлежащих семейству 3 по классификации [20]):

$$
\boldsymbol{x}(\boldsymbol{X})=e_{R} \sqrt{\left(\boldsymbol{e}^{R} \cdot \boldsymbol{X}\right)^{2}+a}+e_{Z} \otimes e^{Z} \cdot \boldsymbol{X}
$$

где $a$ - параметр деформации, определяющий изменение радиуса внешней цилиндрической поверхности тела-слоя. Соответствующие градиент деформации ${ }^{2} \boldsymbol{F}$ и левый тензор Коши - Грина $\boldsymbol{B}=\boldsymbol{F} \cdot \boldsymbol{F} *$ (здесь и далее символ * обозначает операцию транспонирования) определяются разложениями по элементам локального базиса, соответствующего отсчетной позиции точки:

$$
\begin{gathered}
\boldsymbol{F}=\frac{R}{\sqrt{R^{2}+a}} \boldsymbol{e}_{R} \otimes \boldsymbol{e}_{R}+\frac{\sqrt{R^{2}+a}}{R^{3}} \boldsymbol{e}_{\Theta} \otimes \boldsymbol{e}_{\Theta}+\boldsymbol{e}_{Z} \otimes \boldsymbol{e}_{Z}, \\
\boldsymbol{B}=\boldsymbol{F} \cdot \boldsymbol{F}^{*}=\frac{R^{2}}{R^{2}+a} \boldsymbol{e}_{R} \otimes \boldsymbol{e}_{R}+\frac{R^{2}+a}{R^{4}} \boldsymbol{e}_{\Theta} \otimes \boldsymbol{e}_{\Theta}+\boldsymbol{e}_{Z} \otimes \boldsymbol{e}_{Z}
\end{gathered}
$$

Разложения тензора $\boldsymbol{B}$ и обратного к нему $\boldsymbol{B}^{-1}$ по элементам локального базиса $\left\{\boldsymbol{e}^{r}, \boldsymbol{e}^{\theta}, \boldsymbol{e}^{z}\right\}$, соответствующего актуальной позиции точки $\boldsymbol{x}(\boldsymbol{X})$, т. е.

$$
e_{R}=e_{r}, \quad e_{\Theta}=\frac{\sqrt{r^{2}-a}}{r} \quad e_{\theta}, e_{Z}=e_{z}
$$

имеют вид

$$
\begin{gathered}
\boldsymbol{B}=\frac{r^{2}-a}{r^{2}} \boldsymbol{e}_{r} \otimes \boldsymbol{e}_{r}+\frac{1}{r^{2}-a} \boldsymbol{e}_{\theta} \otimes \boldsymbol{e}_{\theta}+\boldsymbol{e}_{z} \otimes \boldsymbol{e}_{z}, \\
\boldsymbol{B}^{-1}=\frac{r^{2}}{r^{2}-a} \boldsymbol{e}_{r} \otimes \boldsymbol{e}_{r}+\frac{r^{2}-a}{r^{4}} \boldsymbol{e}_{\theta} \otimes \boldsymbol{e}_{\theta}+\boldsymbol{e}_{z} \otimes \boldsymbol{e}_{z} .
\end{gathered}
$$

\footnotetext{
${ }^{2}$ Следуя [20], будем полагать, что градиент деформации $\boldsymbol{F}$ представляет линейный оператор, действующий на свой аргумент слева, т. е.

$$
\boldsymbol{x}(\boldsymbol{X}+\boldsymbol{\Delta})=\boldsymbol{x}(\boldsymbol{X})+\boldsymbol{F} \cdot \boldsymbol{\Delta}+\boldsymbol{o}(\|\boldsymbol{\Delta}\|) .
$$
}


Если цилиндрический слой изготовлен из несжимаемого материала типа Муни - Ривлина, то энергия деформации может быть представлена как линейная функция первого $I_{1}=I_{1}(\boldsymbol{B})$ и второго $I_{2}=I_{2}(\boldsymbol{B})$ инвариантов меры деформаций $\boldsymbol{B}$ :

$$
\begin{gathered}
W\left(I_{1}, I_{2}\right)=C_{1}\left(I_{1}-3\right)+C_{2}\left(I_{2}-3\right), \\
I_{1}=\operatorname{Tr} \boldsymbol{B}=3+\frac{a^{2}}{r^{2}\left(r^{2}-a\right)}, \quad I_{2}=1 / 2\left((\operatorname{Tr} \boldsymbol{B})^{2}-\operatorname{Tr}\left(\boldsymbol{B}^{2}\right)\right)=I_{1} .
\end{gathered}
$$

Здесь $C_{1}, C_{2}$ - экспериментально определяемые константы материала. Тензор напряжений Коши, определяемый для несжимаемой среды законом состояния $\boldsymbol{T}=\partial W / \partial B$, может быть определен с точностью до гидростатического давления $p$ формулой [8]:

$$
\boldsymbol{T}=-p \boldsymbol{I}+J_{1} \boldsymbol{B}+J_{-1} \boldsymbol{B}^{-1}
$$

где $\boldsymbol{I}$ - единичный тензор, представляющий тождественное преобразование в $\mathscr{E}, J_{1}=2 \partial W / \partial I_{1}=2 C_{1}$ и $J_{-1}=-2 \partial W / \partial I_{2}=-2 C_{2}-$ коэффициенты реакции материала. Заметим, что константы материала $C_{1}, C_{2}$ могут быть определены через другую пару (инженерных) материальных постоянных $\mu, \beta$ :

$$
C_{1}=\mu(1+\beta) / 4, \quad C_{2}=\mu(1-\beta) / 4 \text {. }
$$

При этом постоянная $\mu$ соответствует модулю сдвига при малых деформациях, а $\beta$ определяет дополнительную параметрическую степень свободы закона состояния. Из термодинамических соображений $-1<\beta<1[20]$.

В результате элементарных вычислений приходим к выражению

$$
\begin{gathered}
\boldsymbol{T}=T^{r r} \boldsymbol{e}_{r} \otimes \boldsymbol{e}_{r}+T^{\theta \theta} \boldsymbol{e}_{\theta} \otimes \boldsymbol{e}_{\theta}+T^{z z} \boldsymbol{e}_{z} \otimes \boldsymbol{e}_{z}, \\
T^{r r}=-p+J_{1} \frac{r^{2}-a}{r^{2}}+J_{-1} \frac{r^{2}}{r^{2}-a}, \quad T^{\theta \theta}=-\frac{p}{r^{2}}+J_{1} \frac{1}{r^{2}-a}+J_{-1} \frac{r^{2}-a}{r^{4}}, \\
T^{z z}=-p+J_{1}+J_{-1} .
\end{gathered}
$$

Гидростатическая компонента напряжений определяется из уравнения равновесия $\nabla \cdot \boldsymbol{T}=\mathbf{0}$, которое в цилиндрических координатах с учетом осевой симметрии имеет вид

$$
\frac{T^{r r}}{r}-r T^{\theta \theta}+\frac{\partial T^{r r}}{\partial r}=0
$$

Решение уравнения (6) может быть записано в квадратурах

$$
T^{r r}=\int\left(r T^{\theta \theta}-\frac{T^{r r}}{r}\right) d r=\int\left(J_{1}-J_{-1}\right)\left(\frac{r}{r^{2}-a}-\frac{r^{2}-a}{r^{3}}\right) d r
$$

Интегрируя равенство (7), с учетом формул (6) будем иметь:

$$
\begin{gathered}
T^{r r}=\frac{\mu}{2}\left(\ln \frac{r^{2}-a}{r^{2}}-\frac{a}{r^{2}}\right)+p_{0}, \quad T^{\theta \theta}=\frac{T^{r r}}{r^{2}}+\frac{\mu}{r^{2}}\left(\frac{r^{2}}{r^{2}-a}-\frac{r^{2}-a}{r^{2}}\right), \\
T^{z z}=T^{r r}+\mu a \frac{r^{2}-(1+\beta) a / 2}{r^{2}\left(r^{2}-a\right)}
\end{gathered}
$$

где $p_{0}-$ постоянная интегрирования. Заметим, что в физическом базисе $\mathbf{e}_{\langle r\rangle}=\mathbf{e}_{r}, \mathbf{e}_{\langle\theta\rangle}=\mathbf{e}_{\theta} / r$, $\mathbf{e}_{\langle z\rangle}=\mathbf{e}_{z}$ компоненты напряжений имеют вид

$$
T_{\langle r r\rangle}=T^{r r}, \quad T_{\langle\theta \theta\rangle}=T^{\theta \theta} r^{2}, \quad T_{\langle z z\rangle}=T^{z z} .
$$

Замечание 1. Деформации вида (4) принадлежат к классу контролируемых деформаций: они могут быть реализованы, если к граничным поверхностям приложить специальным образом подобранные поверхностные силы. 
Ввиду того что деформации заданы с точностью до параметра $a$, а поле напряжений с точностью до постоянной $p_{0}$, возможно точно удовлетворить граничные условия на цилиндрических поверхностях, если на них задана постоянная гидростатическая нагрузка интенсивностью $p_{i}$ и $p_{e}$ :

$$
\left.\boldsymbol{T} \cdot \boldsymbol{e}^{r}\right|_{r=r_{i}}=p_{i} \boldsymbol{e}_{r},\left.\quad \boldsymbol{T} \cdot \boldsymbol{e}^{r}\right|_{r=r_{e}}=p_{e} \boldsymbol{e}_{r},
$$

где $r_{i}, r_{e}$ - радиусы внутренней (internis) и внешней (exterius) цилиндрических граничных поверхностей.

Подстановка выражений для радиальных компонент напряжений (8) в граничные условия (9) с учетом кинематических соотношений (4) приводит к системе двух уравнений

$$
\left\{\begin{array}{l}
\frac{\mu}{2}\left(\ln \frac{R_{i}^{2}}{R_{i}^{2}+a}-\frac{a}{R_{i}^{2}+a}\right)+p_{0}=p_{i}, \\
\frac{\mu}{2}\left(\ln \frac{R_{e}^{2}}{R_{e}^{2}+a}-\frac{a}{R_{e}^{2}+a}\right)+p_{0}=p_{e},
\end{array}\right.
$$

где $R_{i}=\sqrt{r_{i}^{2}-a}, R_{e}=\sqrt{r_{e}^{2}-a}$ - отсчетные значения радиусов граничных поверхностей. Исключая $p_{0}$ из полученной системы, приходим к уравнению относительно параметра деформации $a$ :

$$
\ln \left(\frac{R_{i}^{2}}{R_{e}^{2}} \frac{R_{e}^{2}+a}{R_{i}^{2}+a}\right)=2 \frac{p_{i}-p_{e}}{\mu}+a \frac{R_{e}^{2}-R_{i}^{2}}{\left(R_{i}^{2}+a\right)\left(R_{e}^{2}+a\right)} .
$$

Вводя новую переменную - относительный параметр деформации $x=a / R_{e}^{2}-$ и потенцируя левую и правую части полученного выражения, получим уравнение в виде

$$
F=0, \quad F=\frac{1+x}{\gamma+x}-\frac{A}{\gamma} e^{x \frac{1-\gamma}{(1+x)(\gamma+x)}} .
$$

Здесь $\gamma=R_{i}^{2} / R_{e}^{2}, A=\exp \left[2\left(p_{i}-p_{e}\right) / \mu\right]$. Поскольку внутренний отсчетный радиус не может превышать внешнего и является положительной величиной, то $0<\gamma<1$. Кроме того, для того чтобы под знаком корня в выражении для отсчетного значения радиуса внутренней граничной поверхности была положительная величина, следует потребовать выполнения неравенства $x>-\gamma$. Из предельных соотношений

$$
\lim _{x \rightarrow-\gamma} F=\infty, \quad \lim _{x \rightarrow \infty} F=1-\frac{A}{\gamma}
$$

следует, что уравнение (10) имеет решение при условии $A>\gamma$, т. е. для заданной величины $\gamma$ имеется ограничение для разности интенсивностей гидростатической нагрузки: $p_{i}-p_{e}>\mu / 2 \ln \gamma$.

Замечание 2. Решение трансцендентного уравнения (10) можно найти аналитически с помощью метода обращения степенного ряда (метода Лагранжа). Для этого уравнение (10) разрешается относительно параметра $A$ :

$$
A=\gamma \frac{1+x}{\gamma+x} e^{x \frac{\gamma-1}{(1+x)(\gamma+x)}}
$$

и полученное выражение раскладывается в ряд Тейлора в окрестностях точек $x_{0}=1$ и бесконечно удаленной точки. Для формулировки общих членов разложений в аналитическом виде воспользуемся формулой Лейбница для производной произведения и формулой Фаа-ди-Бруно для производной $n$-го порядка сложной функции:

$$
\frac{d^{n}}{d x^{n}} f(g(x))=\sum_{k=1}^{n} f^{(k)}(g(x)) B_{n, k}\left(g^{\prime}(x), g^{\prime \prime}(x), \ldots, g^{(n-k+1)}(x)\right) .
$$

Здесь $B_{n, k}-$ полиномы Белла

$$
B_{n, k}\left(x_{1}, x_{2}, \ldots, x_{n-k+1}\right)=\sum \frac{n !}{J_{1} ! J_{2} ! \ldots J_{n-k+1} !}\left(\frac{x_{1}}{1 !}\right)^{J_{1}}\left(\frac{x_{2}}{2 !}\right)^{J_{2}} \ldots\left(\frac{x_{n-k+1}}{(n-k+1) !}\right)^{J_{n-k+1}},
$$

при вычислении которых сумма берется по всем последовательностям $J_{1}, J_{2}, \ldots J_{n-k+1}$ неотрицательных целых чисел таких, что

$$
J_{1}+J_{2}+\ldots+J_{n-k+1}=k, \quad J_{1}+2 J_{2}+3 J_{3}+\ldots+(n-k+1) J_{n-k+1}=n .
$$


В результате разложения приходим к следующим формулам:

$$
\begin{aligned}
& A=\sum_{p=0}^{\infty} A_{p}^{1} \frac{(x-1)^{p}}{p !} \text { при }-\gamma<x<2+\gamma, \quad A=\sum_{p=0}^{\infty} A_{p}^{\infty} \frac{1}{x^{p} p !} \text { при } 1<x<\infty, \\
& A_{p}^{1}=e^{\frac{\gamma-1}{2(\gamma+1)}} \sum_{k=0}^{n} q_{n, k}^{1} \sum_{m=0}^{k} B_{k, m}\left(x_{1}^{1}, \ldots, x_{n-k+1}^{1}\right), \quad A_{p}^{\infty}=\sum_{k=0}^{n} q_{n, k}^{\infty} \sum_{m=0}^{k} B_{k, m}\left(x_{1}^{\infty}, \ldots, x_{n-k+1}^{\infty}\right), \\
& x_{p}^{1}=(-1)^{p} p !\left(\gamma(\gamma+1)^{-p-1}-2^{-p-1}\right), \quad x_{p}^{\infty}=(-1)^{p} p !\left(1-\gamma^{p}\right), \\
& q_{n, k}^{1}=(-1)^{n-k} \frac{n !}{k !} \begin{cases}\gamma(1-\gamma)(\gamma+1)^{k-n-1}, & n-k>0, \\
2 \gamma(\gamma+1)^{-1}, & n-k=0,\end{cases} \\
& q_{n, k}^{\infty}=(-1)^{n-k} \frac{n !}{k !} \begin{cases}\gamma^{n-k}(\gamma-1), & n-k>0 \\
\gamma, & n-k=0\end{cases}
\end{aligned}
$$

Величины первых членов прямых разложений (11) отличны от нуля, что позволяет воспользоваться процедурой обращения степенных рядов [23] и выразить относительный параметр деформации $x$ в форме аналитической зависимости от параметра $A$.

По найденному $x$ определяется абсолютный параметр деформации $a=R_{e}^{1} x$ и соответствующее значение $p_{0}$. Таким образом, для заданных значений гидростатических нагрузок $P_{i}, P_{e}$ и отсчетных радиусов граничных поверхностей $R_{i}, R_{e}$ определены параметры $a, p$ и соответственно закон деформирования (4), компоненты тензоров деформации (5) и напряжений (8).

В дальнейшем понадобится выражение для напряжений на поверхностях $\theta=$ const. Соответствующий этим напряжениям главный вектор, который будем называть вектором натяга, имеет следующее выражение:

$$
\boldsymbol{F}=\boldsymbol{e}_{\langle\theta\rangle} \int_{0}^{h} \int_{r_{i}}^{r_{e}} T_{\langle\theta \theta\rangle} d r=\boldsymbol{e}_{\langle\theta\rangle} h\left(\left.r_{e} T_{\langle r r\rangle}\right|_{r=r_{e}}-\left.r_{i} T_{\langle r r\rangle}\right|_{r=r_{i}}\right)
$$

\section{3. ДИСКРЕТНОЕ НАРАЩИВАНИЕ}

Пусть имеются конечные множества тел, которые в натуральной конфигурации представляют собой полые цилиндры с одинаковой высотой $h$, деформируемые преобразованием (4) в полые цилиндры той же высоты, но другой толщины. Такая деформация может быть реализована, например, при расширении полого цилиндра, основания которого лежат на полых гладких плитах. Будем полагать, что образы актуальных конфигураций тел этого множества попарно не пересекаются, а их объединение представляет связное множество - полый цилиндр. Этим определяется геометрически идеальный контакт тел по цилиндрическим поверхностям. Полученное в результате составное тело можно рассматривать как результат дискретного наращивания, если после соединения слоев возникают связи, препятствующие независимому деформированию этих тел-слоев.

Пусть имеется $N$ тел-слоев. Предположим, что реализуется следующий сценарий роста. Осуществляется соединение 1-го тела-слоя со вторым, в результате чего образуется составное тело, которое будем называть первой сборкой. Затем присоединяется 3-е тело-слой, в результате чего получается 2 -я сборка и т.д. На внутренней $r=r_{i, n}^{1}$ и внешней границах $r=r_{e, n}^{n}$ растущего тела, состоящего из $n$ тел-слоев $(n \leq N)$, заданы равномерно распределенные давления $p_{i, n}$ и $p_{e, n}$ :

$$
\left.\boldsymbol{T} \cdot \boldsymbol{e}^{r}\right|_{r=r_{i, n}^{1}}=p_{i, n} \boldsymbol{e}_{r},\left.\quad \boldsymbol{T} \cdot \boldsymbol{e}^{r}\right|_{r=r_{e, n}^{n}}=p_{e, n} \boldsymbol{e}_{r}
$$

Индекс $n$ после запятой указывает на номер сборки, а индексация в обозначениях интенсивности гидростатических нагрузок - на то, что они, вообще говоря, изменяются при переходе от одной сборки к другой. Полагаем, что тела-слои находятся в идеальном контакте, т. е. внутренняя поверхность $k$-го слоя и внешняя поверхность $k+1$-го слоя в образе актуальной конфигурации совпадают, а напряжения на них самоуравновешены, т.е

$$
\left.\boldsymbol{T} \cdot \boldsymbol{e}^{r}\right|_{r=r_{e, n}^{k}}=\left.\boldsymbol{T} \cdot \boldsymbol{e}^{r}\right|_{r=r_{i, n}^{k+1}}, \quad r_{e, n}^{k}=r_{i, n}^{k+1}, \quad k=1,2, \ldots, n-1 .
$$


Параметры деформации $a_{n}^{k}$ и параметры $p_{0, n}^{k}, k=1,2, \ldots, n$ для каждого тела-слоя находятся из системы $2 n$ нелинейных уравнений (13), (14). С учетом (4), (8) эта система принимает вид

$$
\left\{\begin{array}{l}
\frac{\mu}{2}\left[\ln \frac{\left(R_{i}^{1}\right)^{2}}{\left(R_{i}^{1}\right)^{2}+a_{n}^{1}}-\frac{a_{n}^{1}}{\left(R_{i}^{1}\right)^{2}+a_{n}^{1}}\right]+p_{0, n}^{1}=p_{i, n}, \quad \frac{\mu}{2}\left[\ln \frac{\left(R_{e}^{n}\right)^{2}}{\left(R_{e}^{n}\right)^{2}+a_{n}^{n}}-\frac{a_{n}^{n}}{\left(R_{e}^{n}\right)^{2}+a_{n}^{n}}\right]+p_{0, n}^{n}=p_{e, n} \\
\frac{\mu}{2}\left[\ln \frac{\left(R_{e}^{k}\right)^{2}}{\left(R_{e}^{k}\right)^{2}+a_{n}^{k}}-\frac{a_{n}^{k}}{\left(R_{e}^{k}\right)^{2}+a_{n}^{k}}\right]+p_{0, n}^{k}=\frac{\mu}{2}\left[\ln \frac{\left(R_{i}^{k+1}\right)^{2}}{\left(R_{i}^{k+1}\right)^{2}+a_{n}^{k+1}}-\frac{a_{n}^{k+1}}{\left(R_{i}^{k+1}\right)^{2}+a_{n}^{k+1}}\right]+p_{0, n}^{k+1} \\
\left(R_{e}^{k}\right)^{2}+a_{n}^{k}=\left(R_{i}^{k+1}\right)^{2}+a_{n}^{k+1}, \quad k=1,2, \ldots, n-1 .
\end{array}\right.
$$

Исключение переменных, характеризующих гидростатическую компоненту в каждом слое $p_{0, n}^{1}, p_{0, n}^{2}, \ldots, p_{0, n}^{n}$, дает систему $n$ уравнений относительно переменных $a_{n}^{1}, a_{n}^{2}, \ldots, a_{n}^{n}$, т. е.

$$
\begin{gathered}
\ln \left(\frac{\left(R_{i}^{1}\right)^{2}}{\left(R_{e}^{1}\right)^{2}} \frac{\left(R_{i}^{2}\right)^{2}}{\left(R_{e}^{2}\right)^{2}} \ldots \frac{\left(R_{i}^{n}\right)^{2}}{\left(R_{e}^{n}\right)^{2}} \frac{\left(R_{e}^{1}\right)^{2}+a_{n}^{1}}{\left(R_{i}^{1}\right)^{2}+a_{n}^{1}} \frac{\left(R_{e}^{2}\right)^{2}+a_{n}^{2}}{\left(R_{i}^{2}\right)^{2}+a_{n}^{2}} \ldots \frac{\left(R_{e}^{n}\right)^{2}+a_{n}^{n}}{\left(R_{i}^{n}\right)^{2}+a_{n}^{n}}\right)- \\
-a_{n}^{1}\left(\frac{1}{\left(R_{i}^{1}\right)^{2}+a_{n}^{1}}-\frac{1}{\left(R_{e}^{1}\right)^{2}+a_{n}^{1}}\right)-\ldots-a_{n}^{n}\left(\frac{1}{\left(R_{i}^{n}\right)^{2}+a_{n}^{n}}-\frac{1}{\left(R_{e}^{n}\right)^{2}+a_{n}^{n}}\right)=2 \frac{p_{i, n}-p_{e, n}}{\mu} \\
a_{n}^{k}=a_{n}^{1}+\sum_{p=2}^{k}\left(\left(R_{e}^{p-1}\right)^{2}-\left(R_{i}^{p}\right)^{2}\right), \quad k=2,3, \ldots, n .
\end{gathered}
$$

Исключение переменных $a_{n}^{2}, a_{n}^{3}, \ldots, a_{n}^{n}$ приводит к одному уравнению относительно $a_{n}^{1}$

$$
\ln \left[\prod_{k=1}^{n}\left(\gamma_{k} \frac{1+\left(a_{n}^{1}+A_{k}\right) /\left(R_{e}^{k}\right)^{2}}{\gamma_{k}+\left(a_{n}^{1}+A_{k}\right) /\left(R_{e}^{k}\right)^{2}}\right)\right]-\sum_{k=1}^{n} \frac{\left[\left(R_{e}^{k}\right)^{2}-\left(R_{i}^{k}\right)^{2}\right]\left(a_{n}^{1}+A_{k}\right)}{\left(\left(R_{i}^{k}\right)^{2}+a_{n}^{1}+A_{k}\right)\left(\left(R_{e}^{k}\right)^{2}+a_{n}^{1}+A_{k}\right)}=B_{n}
$$

где использованы следующие обозначения:

$$
\gamma_{k}=\left(\frac{R_{i}^{k}}{R_{e}^{k}}\right)^{2}, \quad A_{1}=0, A_{k}=\sum_{p=2}^{k}\left(\left(R_{e}^{p-1}\right)^{2}-\left(R_{i}^{p}\right)^{2}\right), k=2,3, \ldots, n, \quad B_{n}=2 \frac{p_{i, n}-p_{e, n}}{\mu} .
$$

Пусть $\alpha_{k}=1+A_{k} /\left(R_{e}^{k}\right)^{2}, \beta_{k}=\gamma_{k}+A_{k} /\left(R_{e}^{k}\right)^{2}, \nu_{k}=\left(R_{e}^{1}\right)^{2} /\left(R_{e}^{k}\right)^{2}, x_{n}=a_{n}^{1} /\left(R_{e}^{1}\right)^{2}$. Имеем:

$$
\ln \left[\prod_{k=1}^{n} \gamma_{k} \frac{\alpha_{k}+\nu_{k} x_{n}}{\beta_{k}+\nu_{k} x_{n}}\right]-\sum_{k=1}^{n}\left[\left(1-\gamma_{k}\right) \frac{\nu_{k} x_{n}+\alpha_{k}-1}{\left(\beta_{k}+\nu_{k} x_{n}\right)\left(\alpha_{k}+\nu_{k} x_{n}\right)}\right]=B_{n} .
$$

В результате потенцирования левой и правой частей уравнения приходим к следующей его форме:

$$
\prod_{k=1}^{n} \gamma_{k} \frac{\alpha_{k}+\nu_{k} x_{n}}{\beta_{k}+\nu_{k} x_{n}}=W_{n} \exp \left[\sum_{k=1}^{n}\left(1-\gamma_{k}\right) \frac{\nu_{k} x_{n}+\alpha_{k}-1}{\left(\beta_{k}+\nu_{k} x_{n}\right)\left(\alpha_{k}+\nu_{k} x_{n}\right)}\right], \quad W_{n}=e^{2 \frac{p_{i, n}-p_{e, n}}{\mu}} .
$$

Будем различать следующие виды наращивания.

1. Наращивание с заданной отсчетной геометрией. Полагаем, что заданы геометрические характеристики тел-слоев $\mathscr{B}_{k}(k=1, \ldots, N)$, в образе натуральной конфигурации, т. е. отсчетные радиусы ненапряженных тел-слоев $R_{i}^{k}$ и $R_{e}^{k}$.

2. Наращивание с заданной актуальной геометрией. Задано положение растущей границы в образе актуальной конфигурации $\mathscr{R}_{n}$, толщины тел-слоев в образе отсчетной конфигурации, т. е. величины $\Delta^{k}=R_{e}^{k}-R_{i}^{k}, k=1, \ldots, n$.

3. Наращивание с заданым натягом $\mathscr{T}_{n}$. Задано среднее значение окружных напряжений, вычисленных по формуле (12) в каждом теле-слое в момент его присоединения к растущему телу, а также, как и в предыдущем пункте, толщины тел-слоев в образе отсчетной конфигурации.

Рассмотрим каждый из этих типов наращивания подробнее.

Tun 1. Наращивание с заданной отсчетной геометрией. По заданным значениям отсчетных радиусов $R_{i}^{k}, R_{e}^{k}, k=1, \ldots, n$, вычисляются значения $\alpha_{k}, \gamma_{k}, \beta_{k}, \nu_{k}$, а по заданным значениям гидростатической нагрузки $p_{i, n}, p_{e, n}$ вычисляются значения $W_{n}$. В результате приходим к последовательности несвязанных нелинейных уравнений (18), из решения которых независимо определяются 
параметры деформации $x_{n}$. При решении уравнений могут быть использованы представления, указанные в замечании 2. По формулам (17) вычисляются параметры $a_{n}^{k}, k=1, \ldots, n$, а из соотношений (8) определяются напряжения.

Tun 2. Наращивание с заданной актуальной геометрией. В этом случае отсчетные радиусы телслоев заранее не известны, а уравнения (18) дополняются уравнениями, задающими радиус растущей границы $\mathscr{R}_{n}$ в образах промежуточных конфигураций:

$$
r_{e, n}^{n}=\sqrt{\left(R_{e}^{n}\right)^{2}+a_{n}^{n}}=\mathscr{R}_{n} .
$$

Для анализа системы уравнений вначале выделим в левой и правой частях уравнения (18) члены, отвечающие слою с номером $n$ :

$$
\begin{gathered}
\gamma_{n} \frac{\alpha_{n}+\nu_{n} x_{n}}{\beta_{n}+\nu_{n} x_{n}} \prod_{k=1}^{n-1} \gamma_{k} \frac{\alpha_{k}+\nu_{k} x_{n}}{\beta_{k}+\nu_{k} x_{n}}= \\
=W_{n} \exp \left[\frac{\left(1-\gamma_{n}\right)\left(\nu_{n} x_{n}+\alpha_{n}-1\right)}{\left(\beta_{n}+\nu_{n} x_{n}\right)\left(\alpha_{n}+\nu_{n} x_{n}\right)}+\sum_{k=1}^{n-1} \frac{\left(1-\gamma_{k}\right)\left(\nu_{k} x_{n}+\alpha_{k}-1\right)}{\left(\beta_{k}+\nu_{k} x_{n}\right)\left(\alpha_{k}+\nu_{k} x_{n}\right)}\right] .
\end{gathered}
$$

Величины $\gamma_{n}, \alpha_{n}, \beta_{n}, \nu_{n}$, в отличие от наращивания по muny 1 , не могут быть заданы заранее, поскольку они зависят от безразмерного параметра деформации первого слоя $x_{n}$. Действительно, так как

$$
\left(R_{e}^{n}\right)^{2}=\mathscr{R}_{n}^{2}-a_{n}^{n}=\mathscr{R}_{n}^{2}-\left(R_{e}^{1}\right)^{2} x_{n}-A_{n}, \quad R_{i}^{n}=R_{e}^{n}-\Delta^{n},
$$

то выражение для $A_{n}$

$$
A_{n}=A_{n-1}+\left(R_{e}^{n-1}\right)^{2}-\left(R_{i}^{n}\right)^{2}
$$

в действительности оказывается алгебраическим уравнением, решение которого определяет $A_{n}$ через параметры с индексами $m<n$, актуальный радиус растущей границы $\mathscr{R}_{n}$ и параметр деформации $x_{n}$, T. e.

$$
A_{n}=A_{n-1}+\left(R_{e}^{n-1}\right)^{2}-\left(\sqrt{\mathscr{R}_{n}^{2}-\left(R_{e}^{1}\right)^{2} x_{n}-A_{n}}-\Delta^{n}\right)^{2} .
$$

Решение этого уравнения приводит к явному выражению для $A_{n}$ :

$$
A_{n}=\mathscr{R}_{n}^{2}-\left(R_{e}^{1}\right)^{2} x_{n}-\left(\frac{\mathscr{R}_{n}^{2}-A_{n-1}-\left(R_{e}^{n-1}\right)^{2}-\left(R_{e}^{1}\right)^{2} x_{n}+\left(\Delta^{n}\right)^{2}}{2 \Delta^{n}}\right)^{2}
$$

и соответственно к явным выражениям для $\gamma_{n}, \alpha_{n}, \beta_{n}, \nu_{n}$. Имеем:

$$
\begin{gathered}
\gamma_{n}=\left(1-\frac{2\left(\Delta^{n}\right)^{2}}{\mathscr{H}_{n}-\left(R_{e}^{1}\right)^{2} x_{n}}\right)^{2}, \quad \alpha_{n}=1+\nu_{n}\left[\frac{\mathscr{R}_{n}^{2}}{\left(R_{e}^{1}\right)^{2}}-x_{n}-\left(\frac{\mathscr{H}_{n}-\left(R_{e}^{1}\right)^{2} x_{n}}{2 R_{e}^{1} \Delta^{n}}\right)^{2}\right], \\
\beta_{n}=\nu_{n} \frac{A_{n-1}+\left(R_{e}^{n-1}\right)^{2}}{\left(R_{e}^{1}\right)^{2}}, \quad \nu_{n}=4\left(\frac{R_{e}^{1} \Delta^{n}}{\mathscr{H}_{n}-\left(R_{e}^{1}\right)^{2} x_{n}}\right)^{2}, \\
\mathscr{H}_{n}=\mathscr{R}_{n}^{2}-A_{n-1}-\left(R_{e}^{n-1}\right)^{2}+\left(\Delta_{n}\right)^{2} .
\end{gathered}
$$

Подстановка этих выражений в уравнение (19) приводит его к явному виду:

$$
\begin{gathered}
\left(1+\frac{2 \xi_{n}}{\sigma_{n}-x_{n}}\right)^{2} \frac{\zeta_{n}}{\phi_{n-1}+x_{n}} \prod_{k=1}^{n-1} \gamma_{k} \frac{\alpha_{k}+\nu_{k} x_{n}}{\beta_{k}+\nu_{k} x_{n}}= \\
=W_{n} \exp \left[\frac{\sigma_{n}-x_{n}-\xi_{n}}{\phi_{n-1}+x_{n}}\left(1-\frac{\left(\sigma_{n}-x_{n}\right)^{2}}{4 \zeta_{n} \xi_{n}}\right)+\sum_{k=1}^{n-1} \frac{\left(1-\gamma_{k}\right)\left(\nu_{k} x_{n}+\alpha_{k}-1\right)}{\left(\beta_{k}+\nu_{k} x_{n}\right)\left(\alpha_{k}+\nu_{k} x_{n}\right)}\right] .
\end{gathered}
$$

Здесь

$$
\xi_{n}=\left(\frac{\Delta^{n}}{R_{e}^{1}}\right)^{2}, \quad \sigma_{n}=\frac{\mathscr{H}_{n}}{\left(R_{e}^{1}\right)^{2}}, \quad \zeta_{n}=\left(\frac{\mathscr{R}_{n}}{R_{e}^{1}}\right)^{2}, \quad \phi_{n-1}=\frac{A_{n-1}+\left(R_{e}^{n-1}\right)^{2}}{\left(R_{e}^{1}\right)^{2}} .
$$

Решая последовательно эти уравнения, находим параметры $x_{n}$. 
Tun 3. Наращивание с заданным натягом. Известно значение натяга $\mathscr{T}_{n}$ в последнем теле-слое. Согласно формуле (12) это значение определяется так:

$$
\mathscr{T}_{n}=h\left(\left.r T_{\langle r r\rangle}\right|_{r=r_{e, n}^{n}}-\left.r T_{\langle r r\rangle}\right|_{r=r_{i, n}^{n}}\right) .
$$

Заметив, что $\left.T_{\langle r r\rangle}\right|_{r=r_{e, n}^{n}}=p_{n}^{e}$, будем иметь:

$$
\begin{gathered}
\frac{2\left(\mathscr{T}_{n} / h-\left(\sqrt{\left(R_{i}^{n}\right)^{2}+a_{n}^{n}}-\sqrt{\left(R_{e}^{n}\right)^{2}+a_{n}^{n}}\right) p_{e}^{n}\right)}{\mu \sqrt{\left(R_{e}^{n}\right)^{2}+a_{n}^{n}}}=\ln \frac{\left(R_{e}^{n}\right)^{2}\left(\left(R_{i}^{n}\right)^{2}+a_{n}^{n}\right)}{\left(\left(R_{i}^{n}\right)^{2}+a_{n}^{n}\right)\left(R_{i}^{n}\right)^{2}}+ \\
\quad+a_{n}^{n}\left(\frac{1}{\left(R_{i}^{n}\right)^{2}+a_{n}^{n}}-\frac{1}{\left(R_{e}^{n}\right)^{2}+a_{n}^{n}}\right) .
\end{gathered}
$$

В этом случае при каждой $n$-й сборке придется решать следующую систему нелинейных уравнений для определения $R_{0}^{n}, R_{1}^{n}, a_{1, n}$

$$
\left\{\begin{array}{l}
\sum_{k=1}^{n-1}\left[\ln \frac{\left(R_{e}^{k}\right)^{2}\left(\left(R_{i}^{k}\right)^{2}+a_{n}^{k}\right)}{\left(\left(R_{i}^{k}\right)^{2}+a_{n}^{k}\right)\left(R_{i}^{k}\right)^{2}}+a_{n}^{k}\left(\frac{1}{\left(R_{i}^{k}\right)^{2}+a_{n}^{k}}-\frac{1}{\left(R_{e}^{k}\right)^{2}+a_{n}^{k}}\right)\right]+ \\
+\frac{2\left(\mathscr{T}_{n} / h-\left(\sqrt{\left(R_{i}^{k}\right)^{2}+a_{n}^{k}}-\sqrt{\left.\left(R_{e}^{k}\right)^{2}+a_{n}^{k}\right)} p_{e}^{n}\right)\right.}{\mu \sqrt{\left(R_{e}^{k}\right)^{2}+a_{n}^{k}}}=2 \frac{p_{e}^{n}-p_{i}^{n}}{\mu} \\
\frac{2\left(\mathscr{T}_{n} / h-\left(\sqrt{\left(R_{i}^{n}\right)^{2}+a_{n}^{n}}-\sqrt{\left.\left(R_{e}^{n}\right)^{2}+a_{n}^{n}\right)} p_{e}^{n}\right)\right.}{\mu \sqrt{\left(R_{e}^{n}\right)^{2}+a_{n}^{n}}}= \\
=\ln \frac{\left(R_{e}^{n}\right)^{2}\left(\left(R_{i}^{n}\right)^{2}+a_{n}^{n}\right)}{\left(\left(R_{i}^{n}\right)^{2}+a_{n}^{n}\right)\left(R_{i}^{n}\right)^{2}}+a_{n}^{n}\left(\frac{1}{\left(R_{i}^{n}\right)^{2}+a_{n}^{n}}-\frac{1}{\left(R_{e}^{n}\right)^{2}+a_{n}^{n}}\right) .
\end{array}\right.
$$

\section{4. НЕПРЕРЫВНОЕ НАРАЩИВАНИЕ}

С физической точки зрения непрерывное наращивание представляет непрерывный процесс присоединения к растущему телу континуального семейства предварительно деформированных материальных поверхностей $\mathfrak{M}_{\gamma}$. При этом НДС растущего тела зависит как от внешних силовых полей, так и предварительной деформации материальных поверхностей, образующих тело.

Для анализа НДС удобно ввести некоторую промежуточную конфигурацию, образ которой не свободен от напряжений, но его можно погрузить в евклидово пространство. Все величины, отнесенные к промежуточной конфигурации, далее обозначаются символом с тильдой $(\sim)$. В частности, локальный базис, отвечающий положению материальной точки в промежуточной конфигурации $\widetilde{x}(X)$, обозначим символами $\mathbf{e}_{\tilde{r}}, \mathbf{e}_{\tilde{\theta}}, \mathbf{e}_{\tilde{z}}$. Если от этой конфигурации потребовать выполнения дополнительного условия: локальная конфигурация в окрестности любой внутренней точки не изменяется в процессе роста, то полная локальная деформация, трансформирующая окрестность материальной точки из ненапряженного состояния в напряженное соответствует актуальной конфигурации растущего тела, может быть представлена в виде мультипликативной декомпозиции:

$$
\boldsymbol{H}=\boldsymbol{F} \cdot \boldsymbol{K}, \quad \operatorname{rot} \boldsymbol{F}=\mathbf{0}, \quad \operatorname{rot} \boldsymbol{K} \neq \mathbf{0}, \quad \dot{\boldsymbol{K}}=\mathbf{0},
$$

где $\dot{\boldsymbol{K}}$ обозначает производную по времени, либо по времяподобному параметру процесса.

В общем случае промежуточная конфигурация не согласована с актуальными внешними полями, действующими на растущее тело. Это означает, что для реализации этой конфигурации необходимо к телу приложить некоторую систему фиктивных массовых и поверхностных сил, которые имеют характер сил Эшелби [24].

Имея в виду идею о расслоении растущего тела на континуальное семейство материальных поверхностей, каждая из которых в отдельности обладает натуральной, т. е. свободной от напряжений конфигурацией в евклидовом пространстве, эту систему фиктивных сил можно представить континуальным семейством поверхностных нагрузок, удерживающих каждую поверхность в составе образа промежуточной конфигурации. При этом с каждой поверхностью может быть ассоциирована деформация, трансформирующая материальную поверхность из ненапряженного состояния в ее образ в составе промежуточной конфигурации:

$$
\mathbf{K}=\frac{R}{\sqrt{R^{2}+\alpha(R)}} \mathbf{e}_{R} \otimes \mathbf{e}^{R}+\frac{\sqrt{R^{2}+\alpha(R)}}{R} \mathbf{e}_{\Theta} \otimes \mathbf{e}^{\Theta}+\mathbf{e}_{Z} \otimes \mathbf{e}^{Z} .
$$


Заметим, что перенос полей в трехмерное пространство и объединение их в единое поле линейных преобразований порождает гладкое трехмерное поле тензоров второго ранга, которое не является градиентом никакого поля мест, реализуемого в евклидовом пространстве. Кроме того, следует отметить, что в натуральном состоянии множество поверхностей (до деформации полем $\mathbf{K}$ ) не образуют связанного множества в евклидовом пространстве, а после преобразования полем $\mathbf{K}$ образуют связное множество, которое может быть рассмотрено как образ напряженной отсчетной конфигурации. Она подвергается совместной деформации $\mathbf{F}$, имеющей вид

$$
\mathbf{F}=\frac{\widetilde{r}}{\sqrt{\widetilde{r}^{2}+A(t)}} \mathbf{e}_{\widetilde{r}} \otimes \mathbf{e}^{\widetilde{r}}+\frac{\sqrt{\widetilde{r}^{2}+A(t)}}{\widetilde{r}} \mathbf{e}_{\tilde{\theta}} \otimes \mathbf{e}^{\widetilde{\theta}}+\mathbf{e}_{\tilde{z}} \otimes \mathbf{e}^{\widetilde{z}} .
$$

В этом случае полная дисторсия и соответствующее поле деформаций могут быть представлены следующими выражениями:

$$
\begin{gathered}
\mathbf{H}=\mathbf{F} \cdot \mathbf{K}, \quad \mathbf{B}=\mathbf{H} \cdot \mathbf{H}^{*}, \\
\boldsymbol{H}(\widetilde{r}, t)=\frac{\sqrt{\widetilde{r}^{2}-\alpha(\widetilde{r})}}{\sqrt{\widetilde{r}^{2}+A(t)}} \mathbf{e}_{\widetilde{r}} \otimes \mathbf{e}^{\widetilde{r}}+\frac{\sqrt{\widetilde{r}^{2}+A(t)}}{\sqrt{\widetilde{r}^{2}-\alpha(\widetilde{r})}} \mathbf{e}_{\widetilde{\theta}} \otimes \mathbf{e}^{\widetilde{\theta}}+\mathbf{e}_{\tilde{z}} \otimes \mathbf{e}^{\widetilde{z}} .
\end{gathered}
$$

Рассматривая общий случай, будем полагать, что наращивание производится на некоторое начальное тело, которое представляет собой полый цилиндр, свободный от напряжений в начальный момент времени. Его внутренний и внешний радиусы равны соответственно $\widetilde{r}_{i}, \widetilde{r}_{e}$.

Цилиндрические материальные поверхности присоединяются к внешней части тела, непрерывно увеличивая его радиус в промежуточной конфигурации. Текущее значение этого радиуса обозначим $r_{g}$. Пусть на цилиндрических поверхностях растущего тела заданы гидростатические нагрузки $p_{e}$ и $p_{i}$., т. е.

$$
\left.\boldsymbol{T} \cdot \boldsymbol{e}^{\widetilde{r}}\right|_{\widetilde{r}=\widetilde{r}_{i}}=p_{i} \boldsymbol{e}_{\widetilde{r}},\left.\quad \boldsymbol{T} \cdot \boldsymbol{e}^{\widetilde{r}}\right|_{\widetilde{r}=\widetilde{r}_{e}}=p_{e} \boldsymbol{e}_{\widetilde{r}}
$$

Тогда физические компоненты тензора напряжений, вызванные локальной деформацией $\mathbf{H}$, могут быть представлены следующими формулами:

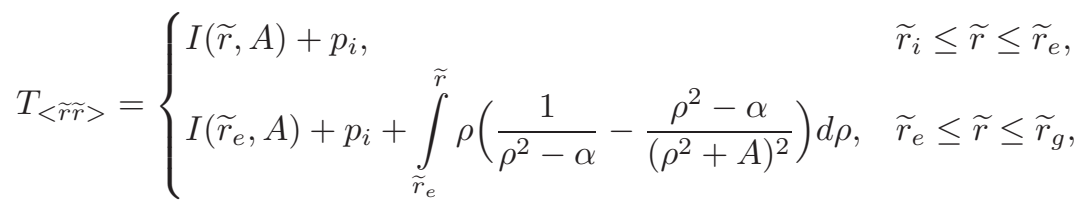

$$
\begin{aligned}
& T_{<\tilde{\theta} \widetilde{\theta}>}=T_{<\widetilde{r} \widetilde{r}>}+ \begin{cases}\frac{\widetilde{r}^{2}+A}{\widetilde{r}^{2}}-\frac{\widetilde{r}^{2}}{\widetilde{r}^{2}+A}, \quad \widetilde{r}_{i} \leq \widetilde{r} \leq \widetilde{r}_{e}, \\
\frac{\widetilde{r}^{2}+A}{\widetilde{r}^{2}-\alpha}-\frac{\widetilde{r}^{2}-\alpha}{\widetilde{r}^{2}+A}, \quad \widetilde{r}_{e} \leq \widetilde{r} \leq \widetilde{r}_{g},\end{cases} \\
& T_{<\tilde{z} \tilde{z}>}=T_{<\widetilde{r} \widetilde{r}}+ \begin{cases}\frac{\widetilde{r}^{2}+A-(1+\beta) A / 2}{\left(\widetilde{r}^{2}+A\right) \widetilde{r}^{2}} A, & \widetilde{r}_{i} \leq \widetilde{r} \leq \widetilde{r}_{e}, \\
\frac{\widetilde{r}^{2}+A-(1+\beta)(A+\alpha) / 2}{\left(\widetilde{r}^{2}+A\right)\left(\widetilde{r}^{2}+\alpha\right)}(A+\alpha), & \widetilde{r}_{e} \leq \widetilde{r} \leq \widetilde{r}_{g},\end{cases} \\
& I(\widetilde{r}, A)=\int_{\widetilde{r}_{i}}^{\widetilde{r}} \rho\left(\frac{1}{\rho^{2}}-\frac{\rho^{2}}{\left(\rho^{2}+A\right)^{2}}\right) d \rho=\ln \frac{\widetilde{r} \sqrt{A+\widetilde{r}_{i}^{2}}}{r_{i} \sqrt{A+\widetilde{r}_{i}^{2}}}-\frac{A}{2}\left(\frac{1}{A+\widetilde{r}_{i}^{2}}+\frac{1}{A+\widetilde{r}_{i}^{2}}\right) .
\end{aligned}
$$

Радиальные напряжения $T_{<\widetilde{r} r>}$ в окрестности растущей границы задаются формулой

$$
T_{<\tilde{r} r}>=I\left(\widetilde{r}_{e}, A\right)+p_{i}+\int_{\widetilde{r}_{e}}^{\widetilde{r}_{g}} \rho\left(\frac{1}{\rho^{2}-\alpha}-\frac{\rho^{2}-\alpha}{\left(\rho^{2}+A\right)^{2}}\right) d \rho .
$$

Окружные напряжения $T_{<\widetilde{\theta} \tilde{\theta}>}$ в окрестности растущей границы могут быть определены соотношением

$$
T_{<\tilde{\theta} \tilde{\theta}>\left.\right|_{\tilde{r}}=\widetilde{r}_{g}}=p_{e}+\frac{\widetilde{r}^{2}+A}{\widetilde{r}^{2}-\alpha\left(\widetilde{r}_{g}\right)}-\frac{\widetilde{r}^{2}-\alpha\left(\widetilde{r}_{g}\right)}{\widetilde{r}^{2}+A} .
$$


Радиус внешней цилиндрической границы в актуальной конфигурации определяется равенством

$$
r_{g}=\sqrt{\widetilde{r}_{g}^{2}+A} .
$$

Темп изменения материального состава тела может быть задан функцией $V(t)$, определяющей закон изменения объема растущего тела в ходе процесса роста. В силу несжимаемости материала она инвариантна относительно перехода к различным конфигурациям. В предположении, что внутренний радиус $\widetilde{r}_{i}$ не изменяется во времени, радиус растущей границы $r_{g}$ может быть определен следующим образом:

$$
\widetilde{r}_{g}=\sqrt{V(t) /(\pi h)+\widetilde{r}_{i}^{2}} .
$$

Рассмотрим типы наращивания, аналогичные рассмотренным в случае дискретного наращивания. Считаем, что во всех случаях известен объем тела, т. е. $V(t)$, а также начальные размеры начальной части.

Tun 1. Наращивание с заданной дисторсией. Задается функция дисторсии $\alpha=\alpha(\widetilde{r})$. Для определения поля напряжений требуется найти параметр $A(t)$, который определяется неявно уравнением

$$
F(A)=\Delta p, \quad F(A)=I\left(\widetilde{r}_{e}, A\right)+\int_{\widetilde{r}_{e}}^{\widetilde{r}_{g}} \rho\left(\frac{1}{\rho^{2}-\alpha}-\frac{\rho^{2}-\alpha}{\left(\rho^{2}+A\right)^{2}}\right) d \rho .
$$

Здесь $\Delta p=\left(p_{e}-p_{i}\right) / \mu$. При заданной зависимости $\alpha(\widetilde{r})$ и найденной зависимости $A(t)$ напряжения, возникающие в растущем теле в процессе роста, определяются соотношением (23).

Tun 2. Наращивание с заданной поверхностью роста. Задано положение образа границы роста в актуальной конфигурации. В отношении к рассматриваемому примеру это означает, что задана функция $z=r_{g}(t)$. Поскольку радиальная координата растущей границы в промежуточной конфигурации задается соотношением (27), то параметр $A$ определяется из уравнения (26), т. е.

$$
A=z^{2}-\widetilde{r}_{g}^{2} .
$$

Подстановка выражений (29) и (24) в краевые условия (22) приводит к интегральному уравнению относительно $\alpha(\widetilde{r})$ т. е.

$$
\int_{\widetilde{r}_{i}}^{x} \rho\left(\frac{1}{\rho^{2}-\alpha}-\frac{\rho^{2}-\alpha(\rho)}{\left(\rho^{2}+z^{2}(x)-x^{2}\right)^{2}}\right) d \rho=\Delta p-I\left(\widetilde{r}, z^{2}(x)-x^{2}\right), \Delta p=\left(p_{e}-p_{i}\right) / \mu .
$$

В результате замены переменных $\xi=\rho^{2}, y=\rho^{2}-\alpha(\rho), \zeta=x^{2}$, приходим к интегральному уравнению:

$$
\int_{a}^{\zeta}\left(\frac{1}{y(\xi)}-\frac{y(\xi)}{(\xi-A(\zeta))^{2}}\right) d \xi=Q(\xi)
$$

относительно функции $y(\xi)$ при заданных функциях $A(\zeta)=z^{2}(\sqrt{\xi})-\zeta$ и $Q(\zeta)=2(\Delta p(\sqrt{\xi})-$ $\left.-I\left(\widetilde{r}_{e}, A(\zeta)\right)\right)$.

Решение этого уравнения определяет функцию $\alpha(\rho)$ и, следовательно, все компоненты напряжений (23).

Tun 3. Наращивание с заданным натягом. Задан натяг в присоединяемом слое, т.е в случае непрерывного наращивания известно $T_{\langle\widetilde{\theta} \widetilde{\theta}}(t)$ на поверхности роста.

Из уравнения $\left.T_{<\widetilde{\theta} \tilde{\theta}>}\right|_{r=r_{g}}=\mathscr{T}$ и соотношения (25) вытекает, что алгебраическое соотношение между параметрами $A$ и $\widetilde{r}_{g}$ имеет вид

$$
r_{g}^{2}+A=\frac{\widetilde{r}_{g}^{2}-\alpha\left(\widetilde{r}_{g}\right)}{2}\left(q \pm \sqrt{q^{2}+4}\right), \quad q=\frac{\mathscr{T}-p_{e}}{\mu} .
$$

Поскольку должны выполняться неравенства $\widetilde{r}_{g}^{2}+A \geq 0, \widetilde{r}_{g}^{2}-\alpha \geq 0$, то в выражении (31) перед радикалом следует использовать знак «Плюс». Таким образом, имеем:

$$
A=\frac{\widetilde{r}_{g}^{2}-\alpha\left(\widetilde{r}_{g}\right)}{2}\left(q+\sqrt{q^{2}+4}\right)-r_{g}^{2} .
$$


Алгебраическое соотношение (32) и краевое условие (22), в которое следует подставить выражение (24), приводят к интегральному уравнению того же типа, что и (30):

$$
\int_{a}^{\zeta}\left(\frac{1}{y(\xi)}-\frac{y(\xi)}{(\xi-A(\zeta))^{2}}\right) d \xi=Q(\xi), \quad A(\zeta)=\frac{y(\zeta)}{2}\left(q+\sqrt{q^{2}+4}\right)
$$

В результате решения этого уравнения определяется функция $y(\xi)$ и соответственно $\alpha(\rho)=\rho^{2}-y\left(\rho^{2}\right)$. все компоненты напряжений определяются из соотношений (23).

\section{5. ПРИМЕРЫ}

На основе приведенных соотношений были разработаны алгоритмы для вычисления напряженнодеформированного состояния дискретного и непрерывного наращивания. Задачи для дискретного наращивания сводились к последовательностям нелинейных уравнений, которые рекурсивно решались методом Ньютона. Задачи для непрерывного наращивания сводились к нелинейным интегральным уравнениям типа Урысона-Вольтерра, решения которых находились методом квадратур [25]. Ниже приведены некоторые результаты этих вычислений.

Рассматривался резиноподобный материал типа Муни-Ривлина, механические характеристики которого взяты из [26], а именно $J_{1}=9.83810^{5}$ Па, $J_{-1}=-6.01710^{5}$ Па. Этим значениям соответствуют «инженерные» константы: $\mu=7.92810^{5}$ Па, $\beta=0.241$. Во всех вычислениях внешний и внутренний радиусы начального тела принимались равными $R_{i}^{1}=1.8 h, R_{e}^{1}=2 h$. В случае дискретного наращивания рассматривались два сценария, в ходе которых присоединялись 5, 10 и 25 слоев соответственно. Толщины их подбирались таким образом, что финальный объем растущего тела оказывался одинаковым. Давление на внутренней и внешней поверхностях наращиваемого тела принималось равное нулю. Момент времени окончания роста $T$ для непрерывного наращивания и толщины слоев в случае дискретного наращивания подбирались так, что объем тела увеличивался вдвое.

Для всех видов непрерывного наращивания принимался следующий закон эволюции растущей границы в образе промежуточной конфигурации:

$$
\widetilde{r}_{i}=1.8, \quad \widetilde{r}_{e}(t)=2+t .
$$

Этому закону соответствует значение $T=0.182$ с.

В случае наращивания с заданной дисторсией принималось, что

$$
\alpha(\widetilde{r})=\left\{\begin{array}{l}
1+\widetilde{r}, \widetilde{r}_{e}(0) \leq \widetilde{r} \leq \widetilde{r}_{e}(t), \\
0, \widetilde{r}_{i}<\widetilde{r} \leq \widetilde{r}_{e}(0)
\end{array}\right.
$$

Дискретное и непрерывное наращивания согласовывались следующим образом. Интервал $\left[\widetilde{r}_{e}, \widetilde{r}_{g}\right]$, соответствующий приращению радиуса внешней цилиндрической поверхности в процессе наращивания, заданного в образе промежуточной конфигурации, разбивался на одинаковые интервалы. Рассматривалось три сценария дискретного наращивания, соответствующих 5, 10 и 25 подынтервалам. При сопоставлении дискретных и непрерывных режимов роста с заданной дисторсией отсчетные радиусы тел слоев выбирались таким образом, что соответствующий им параметр дисторсии $a_{n}$, трансформирующий тело-слой в образ промежуточной конфигурации, численно был равен значению $\alpha\left[\left(\widetilde{r}_{e}^{n}-\widetilde{r}_{i}^{n}\right) / 2\right]$. Графики распределения относительных радиальных окружных и осевых напряжений приведены на рис. 1-3 (a). Точечный пунктир соответствует непрерывному наращиванию, а сплошные линии дискретному наращиванию при $N=5$. На рис. 1-3 (б) показаны различия в значениях напряжений, соответствующих непрерывному и дискретному режимам (штриховой пунктир $N=5$, точечный пунктир $N=10$, сплошная линия $N=25)$. 


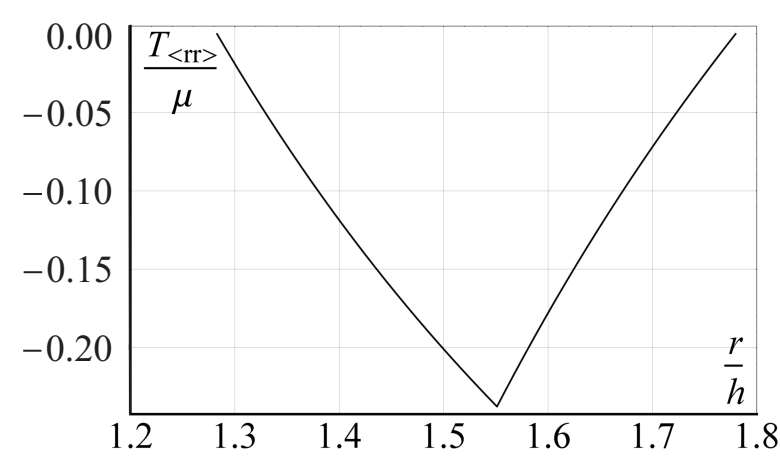

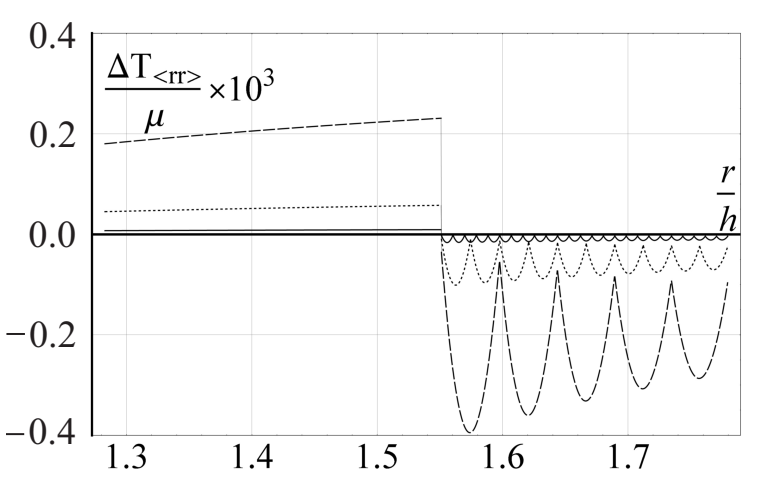

$\sigma$

Рис. 1. Относительные радиальные напряжения при наращивании с заданной дисторсией

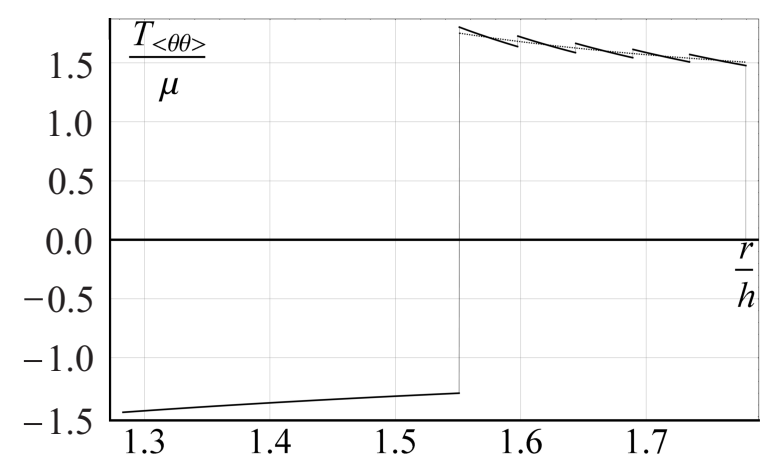

$a$

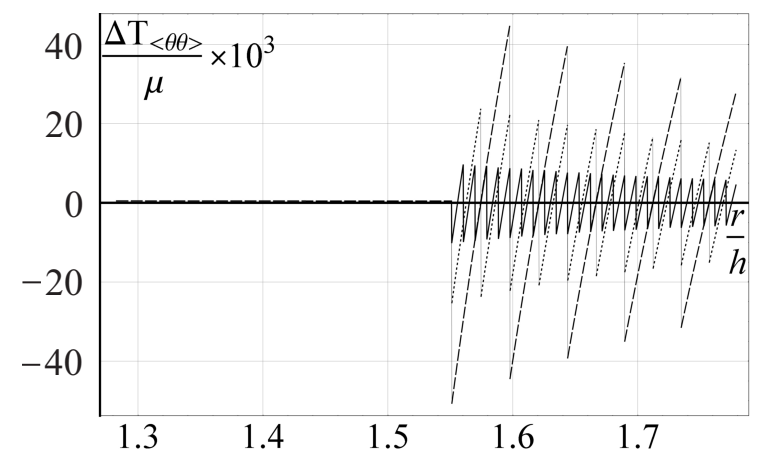

$\sigma$

Рис. 2. Относительные окружные напряжения при наращивании с заданной дисторсией

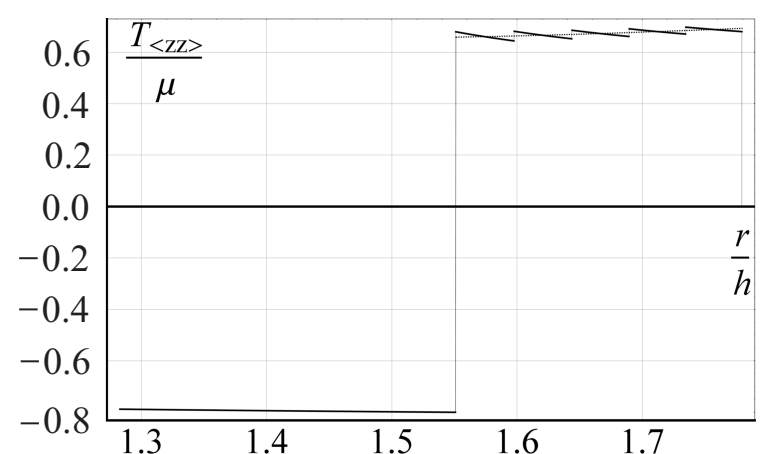

$a$

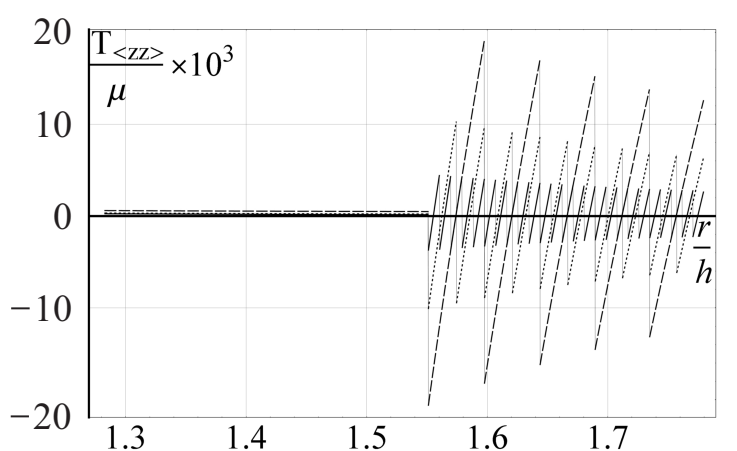

б

Рис. 3. Относительные осевые напряжения при наращивании с заданной дисторсией

В случае непрерывного наращивания с заданной поверхностью роста считался заданным следующий закон движения растущей границы в актуальной конфигурации:

$$
r_{e}(t)=2
$$

В случае соответствующего дискретного наращивания внешние радиусы сборок принимались равными 2h. Рассматривалось три сценария дискретного наращивания, соответствующих присоединению 5,10 и 25 слоев. Графики распределения относительных радиальных окружных и осевых напряжений приведены на рис. 4-6 (a). Различия между непрерывным и дискретным режимами показаны на рис. 4-6 (б). 


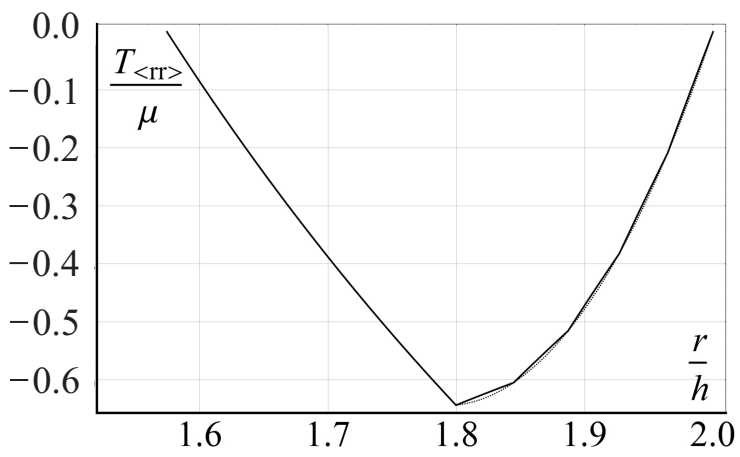

$a$

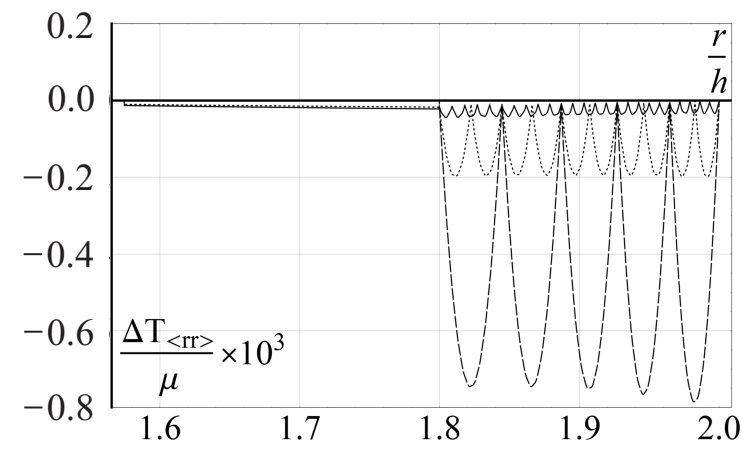

6

Рис. 4. Относительные радиальные напряжения при наращивании с заданной актуальной геометрией

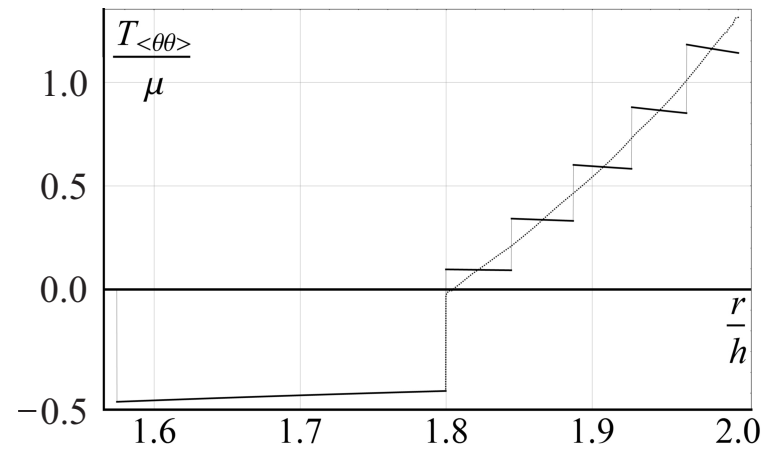

$a$

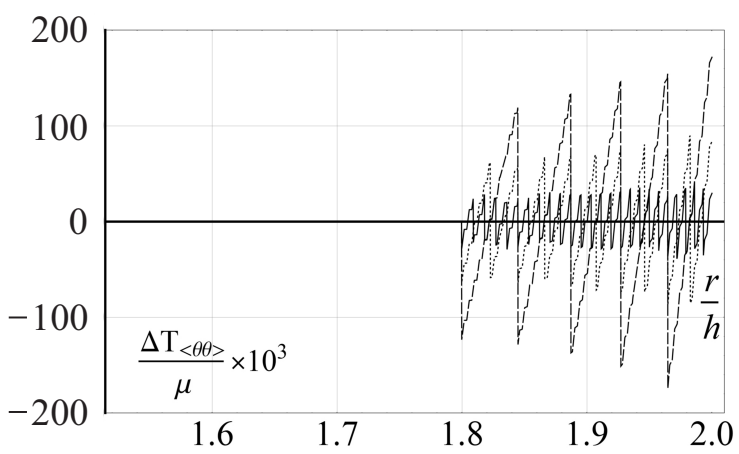

$\sigma$

Рис. 5. Относительные окружные напряжения при наращивании с заданной актуальной геометрией

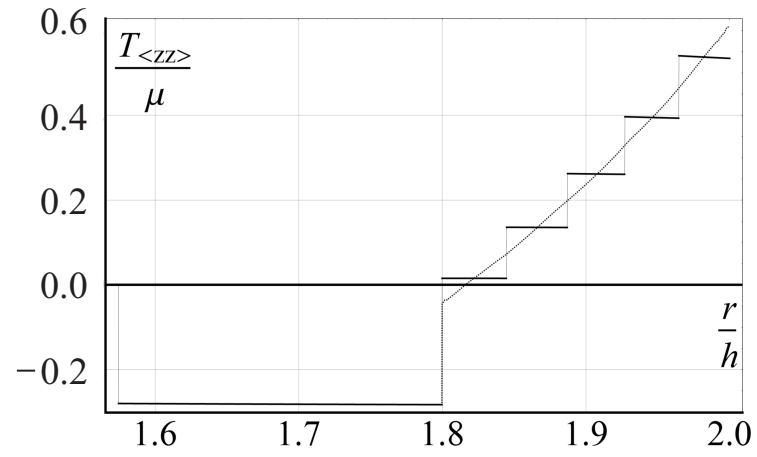

$a$

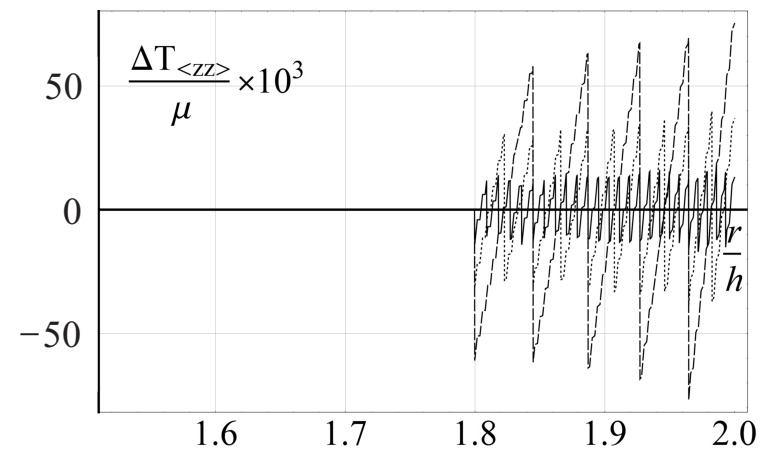

б

Рис. 6. Относительные осевые напряжения при наращивании с заданной актуальной геометрией

В случае непрерывного наращивания с заданным натягом считалось

$$
T_{\langle\theta \theta\rangle}(t)=\mu \text {. }
$$

Соответствующее условие для натяга в дискретных слоях может быть записано в виде

$$
F_{n}=\mu h\left(r_{1, n}^{n}-r_{0, n}^{n}\right),
$$

т.е $T^{\langle\theta \theta\rangle}(t)$ равно среднему окружному напряжению в последнем слое каждой сборки. Графики распределения радиальных окружных и осевых напряжений приведены на рис. 7, $a-8$. Сплошные кривые соответствуют непрерывному наращиванию, «точечная» и пунктирная кривые - дискретному наращиванию при 5 и 10 слоях. 


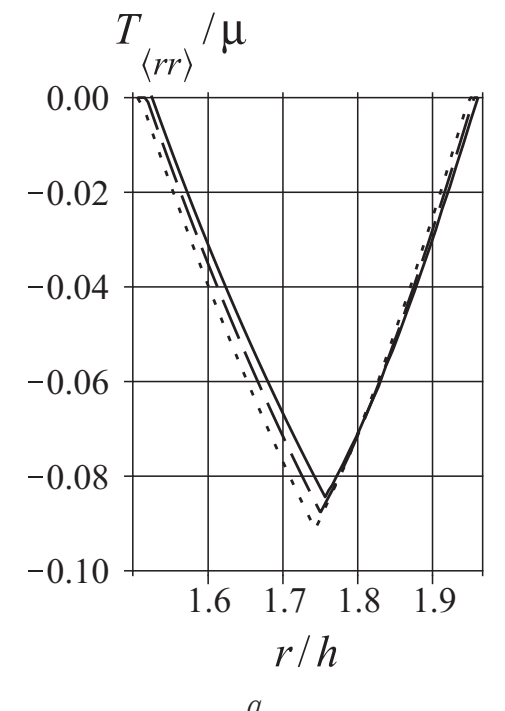

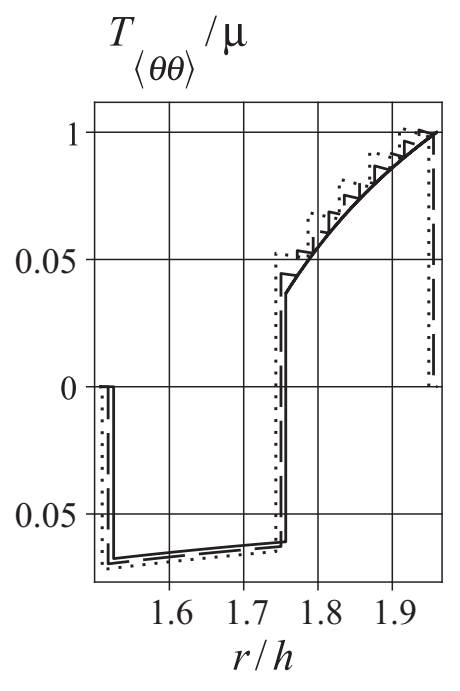

6

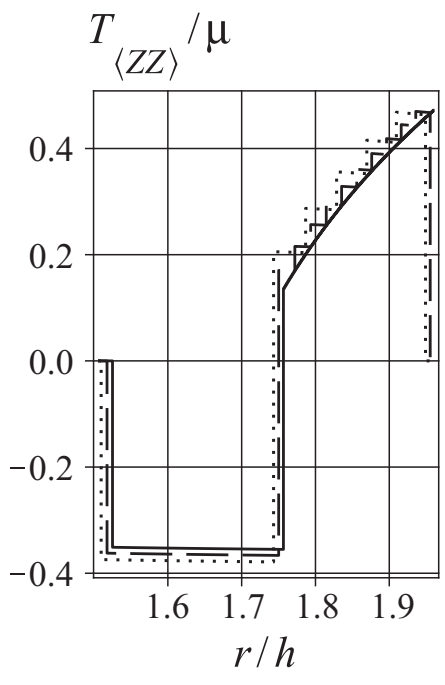

B

Авторы статьи выражают благодарность профессору А. В. Манжирову за конструктивное обсуждение вопросов, отраженных в статье.

Работа выполнена при финансовой поддержке РФФИ (проекты № 12-08-01119-а, № 11-0100669-a).

\section{Библиографический список}

1. Gibson I., Rosen D. W., Stucker B. Additive Manufacturing Technologies. Rapid Prototyping to Direct Digital Manufacturing. Springer, 2009. 459 p.

2. Choy K. L. Chemical vapour deposition of coatings // Progress in Materials Science. 2003. Vol. 48. P. 57-170. 3. Nastasi M., Mayer J. W. Ion Implantation and Synthesis of Materials. Springer, 2006. 263 p.

4. Льчев С. А., Мажжиров А. В. Математическая теория растущих тел. Конечные деформации // ПММ. 2013. Т. 77, вып. 4. С. 585-604.

5. Льчев С. А., Манжиров А. В. Отсчетные конфигурации растущих тел // Изв. РАН. МТТ. 2013. № 5. C. $86-95$.

6. Льчев С. А. Универсальные деформации растущих тел // Изв. РАН. МТТ. 2011. № 6. С. 63-79.

7. Арутюнян Н. Х., Дроздов А. Д., Наумов В. Э. Механика растущих вязкоупругопластичных тел. М. : Наука, 1987. 412 с.

8. Лурье А. И. Нелинейная теория упругости. М. : Наука, 1980. 512 с.

9. Klarbring A., Olsson T., Stalhand J. Theory of residual stresses with application to an arterial geometry // Archives of Mechanics. 2001. Vol. 59. P. 341-364.

10. Epstein M., Maugin G. A. Thermomechanics of volumetric growth in uniform bodies // Intern. J. Plasticity. 2000. Vol. 16, № 7. P. 951-978.

11. Yavari A. A geometric theory of growth mechanics // J. Nonlinear Sci. 2010. Vol. 20, № 6. P. 781-830.

12. Манжиров А. В., Лычев С. А. Математическая теория растущих тел при конечных деформациях // Докл. AH. 2012. T. 443, № 4. C. 438-441.

13. Noll W. Materially uniform simple bodies with inhomogeneities // Arch. Rat. Mech. Anal. 1967. Vol. 27, № 1. P. 1-32.
14. Wang C.-C. On the geometric structures of simple bodies, a mathematical foundation for the theory of continuous distributions of dislocations // Arch. Rat. Mech. Anal. 1967. Vol. 27, № 1. P. 33-94.

15. Epstein M. The Geometrical Language of Continuum Mechanics. Cambridge Univ. Press, 2010. 312 p.

16. Maugin G. A. Material Inhomogeneities in Elasticity. L. : Chapman and Hall, 1993. 294 p.

17. Marsden J. E., Hughes T. J. R. Mathematical Foundations of Elasticity. N. Y. : Dover Publ., 1994. 556 p. 18. Постников М. М. Гладкие многообразия. М. : Наука, 1987. 478 с.

19. Choquet-Bruhat Y., Dewitt-Morette C., DillardBleick M. Analysis, Manifolds and Physics : in 2 pt. Pt. 1. Basics. Amsterdam : Elsevier, 1982. 660 p.

20. Truesdell C., Noll W. The Non-Linear Field Theories of Mechanics. Springer, 2004. 602 p.

21. Manzhirov A. V., Lychev S. A. Residual Stresses in Growing Bodies // Topical Problems in Solid and Fluid Mechanics. Delhi : Elite Pub. House, 2011. P. 66-79.

22. Gurtin M. E., Murdoch A. I. A Continuum Theory of Elastic Material Surfaces. // Arch. Rat. Mech. Anal. 1975. Vol. 27. P. 291-323.

23. Фихтенгольи Г. М. Курс дифференциального и интегрального исчисления : в 3 т. М. : Физматлит, 2001. T. $2.810 \mathrm{c}$.

24. Эщелби Дж. Континуальная теория дислокаций. М. : Изд-во иностр. лит., 1963. 247 с.

25. Полянин А. Д., Манжиров А. В. Справочник по интегральным уравнениям. М. : Физматлит, 2003. 608 с. 26. Hartman S. Numerical studies on the identification of the material parameters of Rivlin's hyperelasticity using tension-torsion tests // Acta Mechanica. 2001. Vol. 148. P. 129-155. 


\title{
Axisymmetric growth of a hollow hyperelastic cylinder
}

\author{
S. A. Lychev, A. V. Mark
}

Institute for Problems in Mechanics of RAS, 101-1, Vernadskogo ave., 119526, Moscow, Russia, lychevsa@mail.ru, A-V-Mark@yandex.ru

\begin{abstract}
The finite deformations of the growing cylinder fabricated of an incompressible elastic material of Mooney - Rivlin type are under consideration. We assume that the deformations are axisymmetric and constant along the cylinder axis. The discrete and continuous types of growing are studied. The analytical solutions of the corresponding boundary-value problems are derived. The computational examples show the convergence of solutions obtained for the discrete growth to corresponding solutions for continuous growth under the following conditions: the number of discrete plies increases while their thickness decreases such that the final volume of growing solid is fixed.
\end{abstract}

Key words: additive technologies, growing solids, finite deformations, hyperelasticity, continuous growth, discrete growth.

This work was supported by the Russian Foundation for Basic Research (projects no. 12-08-01119-a, no. 11-01-00669-a).

\section{References}

1. Gibson I., Rosen D. W., Stucker B. Additive Manufacturing Technologies. Rapid Prototyping to Direct Digital Manufacturing. Springer, 2009. 459 p.

2. Choy K. L. Chemical vapour deposition of coatings. Progress in Materials Science, 2003, vol. 48, pp. 57170.

3. Nastasi M., Mayer J. W. Ion Implantation and Synthesis of Materials. Springer, 2006. 263 p.

4. Lychev S. A., Manzhirov A. V. The mathematical theory of growing bodies. Finite deformations. J. Appl. Math. Mech., 2013, vol. 77, no. 4, pp. 421-432.

5. Lychev S. A., Manzhirov A. V. Reference Configurations of Growing Bodies. Mech. Solids, 2013, vol. 48, no. 5, pp. 553-560.

6. Lychev S. A. Universal Deformations of Growing Solids. Mech. Solids, 2011, vol. 46, no. 6, pp. 863-876.

7. Arutiunian N. Kh., Drozdov A. D., Naumov V. E. Mekhanica rastushih viazkouprugoplastichnikh tel [Mechanics of viscous, elastic, plastic bodies]. Moscow, Nauka, 1987, 412 p. (in Russian).

8. Lurie A. I. Nonlinear theory of elasticity. Amsterdam, North-Holland, 1990, 617 p. (Rus. ed.: Lurie A. I. Nelininay teoria uprugosti. Moscow, Nauka, 1980, 512 p.) 9. Klarbring A., Olsson T., Stalhand J. Theory of residual stresses with application to an arterial geometry. Archives of Mechanics, 2001, vol. 59, pp. 341-364.

10. Epstein M., Maugin G. A. Thermomechanics of volumetric growth in uniform bodies. Int. J. Plasticity, 2000, vol. 16, no. 7, pp. 951-978.

11. Yavari A. A geometric theory of growth mechanics. $J$. Nonlinear Sci., 2010, vol. 20, no. 6, pp. 781-830.

12. Manzhirov A. V., Lychev S. A. The mathematical theory of growing solids: Finite deformations. Doklady Physics, 2012, vol. 57, no. 4, pp. 160-163.

13. Noll W. Materially uniform simple bodies with inhomogeneities. Arch. Rat. Mech. Anal., 1967, vol. 27, no. 1, pp. 1-32.

14. Wang C.-C. On the geometric structures of simple bodies, a mathematical foundation for the theory of

continuous distributions of dislocations. Arch. Rat. Mech. Anal., 1967, vol. 27, no. 1, pp. 33-94.

15. Epstein M. The Geometrical Language of Continuum Mechanics. Cambridge Univ. Press, 2010, 312 p.

16. Maugin G. A. Material Inhomogeneities in Elasticity. London, Chapman and Hall, 1993, 294 p.

17. Marsden J. E., Hughes T. J. R. Mathematical Foundations of Elasticity. New York, Dover Publ., 1994, $556 \mathrm{p}$.

18. Postnikov M. M. Gladkie mnogoobrazia [Smooth manifolds]. Moscow, Nauka, 1987, 478 p. (in Russian).

19. Choquet-Bruhat Y., Dewitt-Morette C., DillardBleick M. Analysis, Manifolds and Physics : in 2 pt. Pt. 1. Basics. Amsterdam, Elsevier, 1982, 660 p.

20. Truesdell C., Noll W. The Non-Linear Field Theories of Mechanics. Springer, 2004, 602 p.

21. Manzhirov A. V., Lychev S. A. Residual Stresses in Growing Bodies. Topical Problems in Solid and Fluid Mechanics. Delhi : Elite Pub. House, 2011, pp. 66-79.

22. Gurtin M. E., Murdoch A. I. A Continuum Theory of Elastic Material Surfaces. Arch. Rat. Mech. Anal., 1975, vol. 27, pp. 291-323.

23. Fihtengolts G. M. Kurs differentsalnogo $i$ integralnogo ischislenia [A course of differential and integral calculus : in 3 vol.]. Moscow, Fizmatlit, 2001, vol. 2, 810 p. (in Russian).

24. Eshelby J. D. The continuum theory of lattice defects. Solid State Physics, 1956, vol. 3, pp. 79-144;

Eshelby J. D. The Determination of the Elastic Field of an Ellipsoidal Inclusion, and Related Problems. Proc. Roy. Soc., ser. A241, 1957, no. 1226, pp. 376-396;

Eshelby J. D. The elastic field outside an ellipsoidal inclusion. Proc. Roy. Soc., ser. A252, 1959, no. 1271, pp. 561-569;

Eshelby J. D., Frank F. C., Nabarro F. R. N. The equilibrium of linear arrays of dislocations. Phil. Mag., 1951, vol. 42, no. 327, pp. 351-364.

25. Polyanin A. D., Manzhirov A. V. Handbook of Inte- 